\title{
Controlled Flight of a Microrobot Powered by Soft Artificial Muscles
}

\section{Citation}

Chen, Yufeng, Huichan Zhao, Jie Mao, Pakpong Chirarattananon, E Farrell Helbling, Nak-Seung Patrick Hyun, David R. Clarke, and Robert J. Wood. 2019. Controlled Flight of a Microrobot Powered by Soft Artificial Muscles. Nature 575, no. 7782: 324-29.

\section{Permanent link}

http://nrs.harvard.edu/urn-3:HUL.InstRepos:42637228

\section{Terms of Use}

This article was downloaded from Harvard University's DASH repository, and is made available under the terms and conditions applicable to Open Access Policy Articles, as set forth at http:// nrs.harvard.edu/urn-3:HUL.InstRepos:dash.current.terms-of-use\#OAP

\section{Share Your Story}

The Harvard community has made this article openly available.

Please share how this access benefits you. Submit a story. 


\section{The first controlled flight of a microrobot powered by soft artificial muscles}

Yufeng Chen ${ }^{1,2^{*}}$, Huichan Zhao ${ }^{3}$, Jie Mao ${ }^{1,4}$, Pakpong Chirarattananon ${ }^{5}$, E. Farrell Helbling ${ }^{1,2}$, Nak-seung Patrick Hyun ${ }^{1,2}$, David R. Clarke ${ }^{1}$, \& Robert J. Wood ${ }^{1,2^{*}}$

${ }^{1}$ John A. Paulson School of Engineering and Applied Sciences, Harvard University, Cambridge, Massachusetts 02138, USA. ${ }^{2}$ Wyss Institute for Biologically Inspired Engineering, Harvard University, Cambridge, Massachusetts 02138, USA. ${ }^{3}$ Department of Mechanical Engineering, Tsinghua University, Beijing 100084, China. ${ }^{4}$ Department of Chemical and Biochemical Engineering, Zhejiang University, Hangzhou 310027, China. ${ }^{5}$ Department of Mechanical and Biomedical Engineering, City University of Hong Kong, Kowloon, Hong Kong, China.

${ }^{*}$ Corresponding authors

Flying insects capable of navigating in highly cluttered natural environments can withstand inflight collisions because of the combination of their low inertia ${ }^{1}$ and the resilience of their wings $^{2}$,

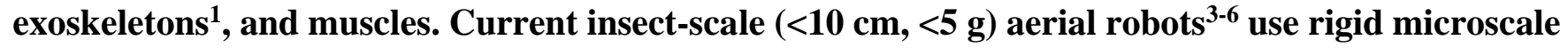
actuators, which are typically fragile under external impact. Biomimetic artificial muscles ${ }^{7-10}$ capable of large deformation offer a promising alternative for actuation because they can endure the stresses caused by such impacts. However, existing soft actuators ${ }^{11-13}$ have not yet demonstrated sufficient power density for liftoff, and their actuation nonlinearity and limited bandwidth further create challenges for achieving closed-loop flight control. Here we develop the first heavier-than-air aerial robots powered by soft artificial muscles that demonstrate open-loop, passively stable ascending flight as well as closed-loop, hovering flight. The robots are driven by 100 mg, multilayered dielectric elastomer actuators (DEA) that have a resonant frequency and power density of $500 \mathrm{~Hz}$ and $600 \mathrm{~W} / \mathrm{kg}$, respectively. To increase actuator output mechanical power and to demonstrate flight control, we present strategies to overcome challenges unique to soft actuators, such as nonlinear transduction and dynamic buckling. These robots can sense, and withstand, collisions with surrounding obstacles, and can recover from in-flight collisions by exploiting material robustness and vehicle passive stability. We further perform a simultaneous flight with 
two micro-aerial-vehicles (MAV) in cluttered environments. These robots rely on offboard amplifiers and an external motion capture system to provide power to the DEAs and control flights. Our work demonstrates how soft actuators can achieve sufficient power density and bandwidth to enable controlled flight, illustrating the vast potential of developing next-generation agile soft robots.

$$
\text { Soft robotics }{ }^{14-16} \text { is an emerging field aiming to develop versatile systems that can safely interact with }
$$
humans and manipulate delicate objects in unstructured environments. A major challenge in building softactuated mobile robots involves developing muscle-like actuators that have high energy density, bandwidth, robustness, and lifetime. Previous studies have described soft actuators that can be actuated chemically $^{17}$, pneumatically ${ }^{18,19}$, hydraulically ${ }^{20}$, thermally ${ }^{21,22}$, or electrically ${ }^{7,23}$. Among these soft transducers, DEAs have shown a combination of muscle-like energy density and bandwidth ${ }^{8}$, enabling the development of biomimetic robots capable of terrestrial ${ }^{11,24,25}$ and aquatic locomotion ${ }^{26,27}$. However, while there is growing interest in developing heavier-than-air, soft-actuated aerial robots, existing soft robots ${ }^{11-}$ ${ }^{13}$ have been unable to achieve liftoff due to limited actuator power density $(<200 \mathrm{~W} / \mathrm{kg})$, bandwidth $(<20$ $\mathrm{Hz}$ ), and difficulties of integration with rigid robotic structures such as transmission and wings.

To enable controlled hovering flight of a soft-actuated robot, we identify and address two major challenges: developing a soft actuator with sufficient power density (>200 W/kg) and designing driving and control strategies to account for actuation nonlinearity (see Methods section 1 for details on vehicle design and DEA performance requirements). First, we develop a multi-layered, compact DEA that has a power density of $600 \mathrm{~W} / \mathrm{kg}$ without requiring pre-strain. Second, we integrate the DEA into a light-weight, flapping wing mechanism and utilize system resonance to remove higher harmonics induced by the nonlinear transduction. In combination, we design a $155 \mathrm{mg}$ flapping-wing module that can be assembled into several configurations. Using these modules, we are able to construct vehicles that not only demonstrate passively stable ascending flight but also controlled hovering flight. 
41 (see Methods section 2 for details on DEA fabrication). The DEA is mounted in a light-weight airframe

42 (Fig. 1a), with the two ends of the DEA attached to planar four-bar transmissions. This design allows one

43 DEA to simultaneously actuate two wings in an analogous manner as the indirect flight muscles in

44 neopteran flying insects ${ }^{28}$. By using the planar four-bar transmissions, the DEA's axial extension and contraction are converted into the wing's rotational stroke motion (Fig. 1b). In quasi-static operation, the actuation is unidirectional because DEA strain is proportional to the square of the applied electric field. In dynamic operation, the DEA extends and contracts due to its intrinsic inertia and stiffness, yet its elongation amplitude is larger than the retraction amplitude. To ensure the mean wing stroke $(\alpha)$ motion is symmetric with respect to the robot body, the resting wing stroke plane is offset by approximately $15^{\circ}$ (Fig. 1b) during robot assembly. The DEA is pre-strained by $2 \%$ when it is attached to the robot transmissions, and this pre-strain loads the elastic four-bar transmissions to introduce the wing stroke bias. This small pre-strain does not noticeably change the DEA performance, and this design is advantageous compared to artificial flight muscles with a large pre-strain ${ }^{11}(>100 \%)$ because it does not require a rigid and heavy supporting structure. In this way, the robot wing stroke $(\alpha)$ motion is fully controlled by the actuator, whereas the wing pitch $(\beta)$ rotation is passively mediated by the compliant wing hinge (Fig. 1c). Figure 1d and Supplementary Video 1 show a half flapping period actuated at $280 \mathrm{~Hz}$. The tracked wing stroke and pitch motion for the same experiment are shown in figure 1e. Based on an aerodynamic model developed in a previous study ${ }^{29}$, we estimate that this flapping motion will generate a net lift force of approximately $1.8 \mathrm{mN}$, corresponding to 1.2 times the robot weight. This modular robot can be assembled into several configurations to demonstrate different flight capabilities. For instance, figure 1f shows microaerial vehicles driven by one (center), two (left), and four DEAs (right). These vehicles exhibit open loop liftoff (one DEA), stable ascending flight (two DEAs), and hovering flight through feedback control (four DEAs), respectively. 
a

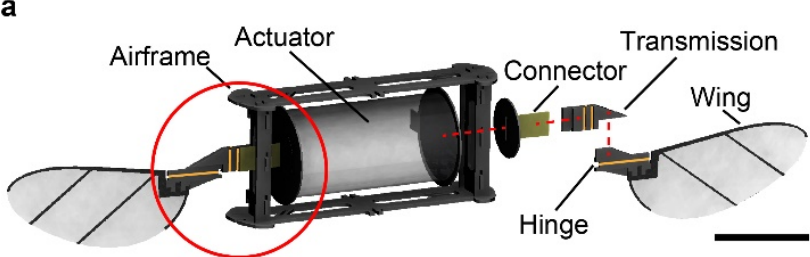

e

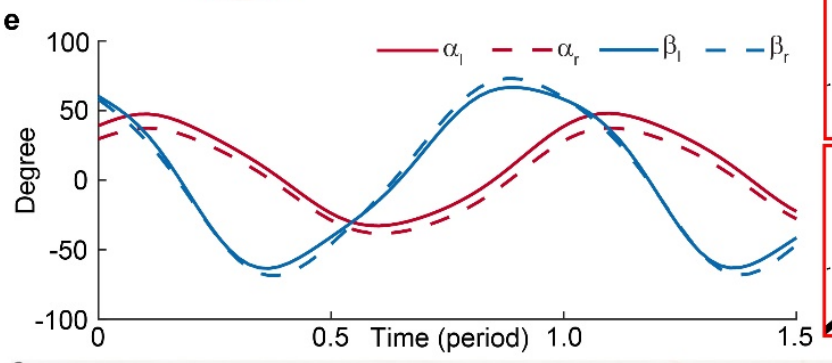

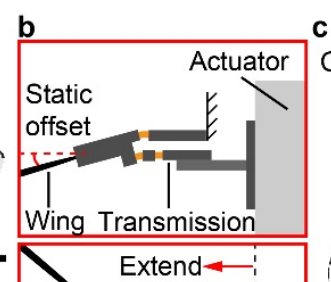
Controll

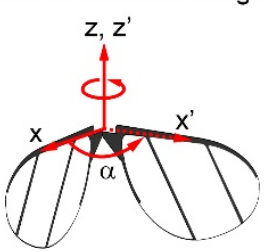

Passive pitch angle

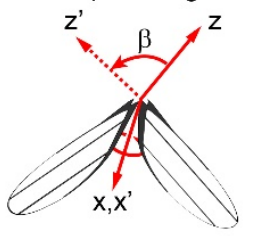

d

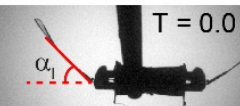

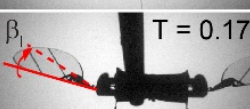

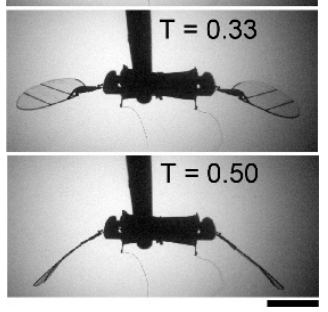

$f$

Figure 1 | Robot design and flapping wing kinematics. a, A CAD model of a $155 \mathrm{mg}$ flapping wing robot driven by a dielectric elastomer actuator (DEA). The exploded view of the robot's right half shows the actuator, connector, four-bar transmission, wing, and wing hinge. The circled region of the robot's left transmission is magnified in $\mathbf{b}$. $\mathbf{b}$, Enlarged top view of the robot's actuator-transmission-wing assembly. The DEA is pre-strained by $2 \%$ when it is attached to the robot's transmissions, which induces a static stroke angle bias of approximately $15^{\circ}$. The linear DEA actuation is translated into the rotational wing stroke motion. c, Illustrations of the actively controlled wing stroke $(\alpha)$ motion and the passive wing pitch $(\beta)$ motion. d, An image sequence of the flapping wing motion operated at $280 \mathrm{~Hz}$. The time is normalized to a flapping period. The wing stroke rotation $\left(\alpha_{l}\right)$ induces passive wing pitch rotation $\left(\beta_{l}\right)$.e, Tracked flapping wing kinematics that correspond to the experiment shown in d. The wing stroke (red) amplitudes of the left (solid line) and the right (dotted line) wings are 42 and 41 degrees, respectively. The wing pitch (blue) amplitudes of the left (solid line) and the right (dotted line) wings are 57 and 61 degrees, respectively. f, Image of flapping wing microrobots driven by a single actuator, two actuators, and four actuators. Scale bars (a, d, f) represent $5 \mathrm{~mm}$.

To achieve flight of a soft-actuated robot, the DEA must have sufficient power density and the robot transmissions and wings must be designed around the actuator's output force, displacement, and bandwidth. In contrast to previous studies ${ }^{11}$ that developed pre-strained acrylic DEAs to achieve large deformation ( $>30 \%$ ) and high energy density $(>4 \mathrm{~J} / \mathrm{kg})$ but low bandwidth $(<30 \mathrm{~Hz})$, we use a silicone elastomer as the dielectric material for the flight muscles to achieve higher bandwidth $(>400 \mathrm{~Hz})$, combined with moderate strain (10-15\%) and energy density $(1.13 \mathrm{~J} / \mathrm{kg})$. For driving frequencies lower than $600 \mathrm{~Hz}$, our DEA's blocked force (Fig. 2a) is independent of frequency because its electrical 
properties are tuned to have a small RC time constant of 0.18 ms. The DEA's free displacement (Fig. 2b) peaks at $15 \%$ strain when it is driven at $500 \mathrm{~Hz}$. The free displacement amplitude includes the contribution from the first and higher order harmonics in response to a sinusoidal driving signal. We observe a secondary peak of free displacement (Fig. 2b) when the driving frequency is $250 \mathrm{~Hz}$, due to exciting the second order harmonic that is near the resonant frequency $(500 \mathrm{~Hz})$. Our robot design utilizes the first harmonic to drive the flapping wing motion. By computing the Fast Fourier Transform (FFT) of the DEA's response to a white noise, we quantify the magnitude (Fig. 2c) and phase (Fig. 2d) of the linear part of its response. When operated at the takeoff condition $(300 \mathrm{~Hz}, 1300 \mathrm{~V})$, the DEA has a power density of 300 $\mathrm{W} / \mathrm{kg}$ and a lifetime of over 600,000 cycles. (see Methods section 3 for details on actuator characterization).

$$
\text { Powering MAVs using soft actuators shows an advantage over the state-of-the-art flapping wing }
$$
microrobots $(<10 \mathrm{~cm},<5 \mathrm{~g})$ driven by rigid actuators such as piezoelectric bimorphs ${ }^{3}$ and electromagnetic motors ${ }^{5}$. Although microrobotic components, such as the airframe, transmissions, and wings, are robust to collisions (because inertial contributions diminish at the millimeter scale), rigid micro-actuators are fragile - particularly the piezoceramic actuators (fracture strength and failure strain are $120 \mathrm{MPa}$ and $0.3 \%$, respectively) used in many similarly sized devices ${ }^{3,4}$. In contrast, this DEA driven microrobot is robust to collisions. For instance, when one wing collides with an obstacle (Fig. 2e and Supplementary Video 2), the impact is absorbed by the DEA because of its high compliance and resilience. In addition, the DEA can detect collisions (Fig. 2f) through concomitant actuation and sensing under similar principles to that of electromagnetic motors ${ }^{30}$ and piezoelectric actuators ${ }^{31}$. Similarly, if an obstacle directly hits the DEA during its actuation (Fig. 2g and Supplementary Video 2), the DEA deformation can also be detected by monitoring the current (Fig. $2 \mathrm{~h}$ ). These experiments show that DEA is not only robust to collisions, but also is capable of sensing collisions with the environment (see Supplementary Information S1 for more experimental results on collision sensing). 
a

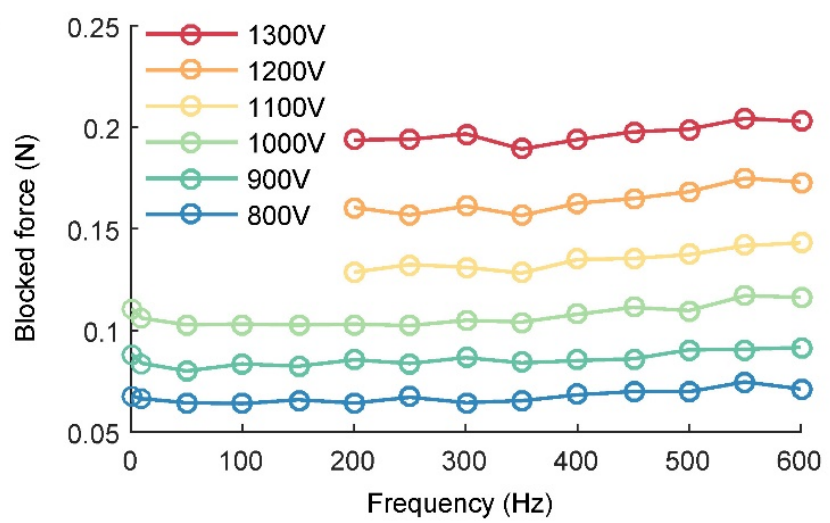

c

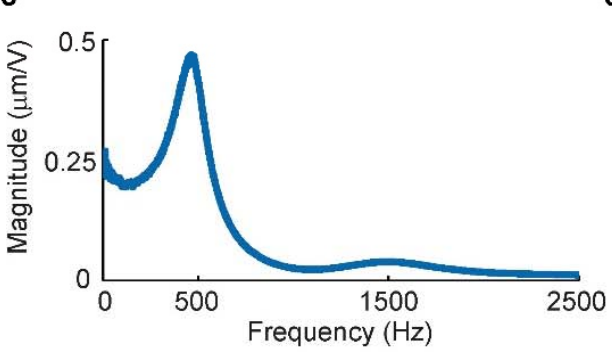

d

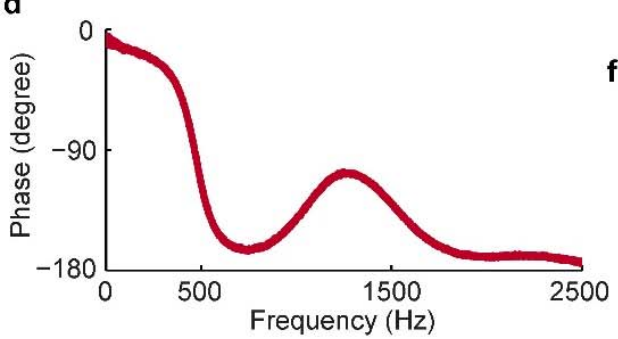

e b
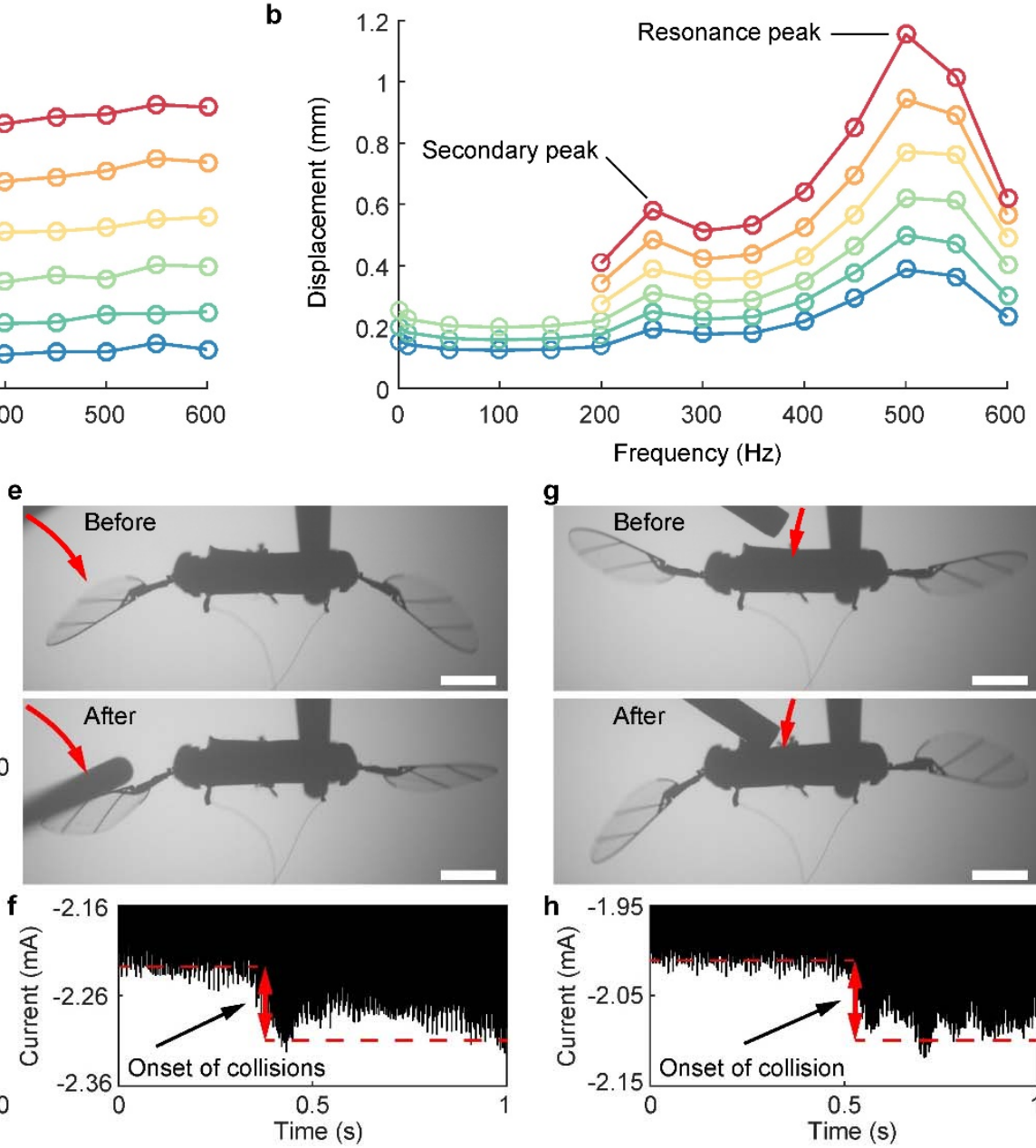

g
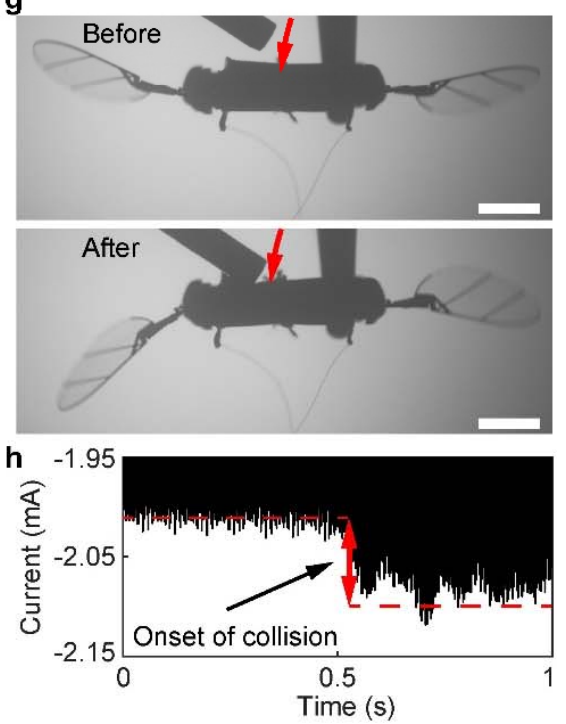

Figure 2 | DEA performance, robustness, and collision sensing. a, b, Measured DEA blocked force (a) and free displacement (b) as functions of operating frequency and voltage amplitude. In $\mathbf{a}$ and $\mathbf{b}$, there are no experiments conducted for the cases combining low frequency $(<200 \mathrm{~Hz})$ and high voltage $(>1000 \mathrm{~V})$ because the elastomer cannot endure a large electric field at low frequencies. c, d, Frequency response of the DEA free displacement under an input voltage of $600 \mathrm{~V}$. c and $\mathbf{d}$ show the magnitude and phase of the frequency response, respectively. e, A flapping wing repeatedly collides with an obstacle when the DEA is operated at $320 \mathrm{~Hz}$ and $1350 \mathrm{~V}$. f, Measured DEA current as a function of time. The jump in the DEA current indicates the onset of the wing-obstacle collisions. g, A rigid object presses down on the DEA that is operating at $320 \mathrm{~Hz}$ and $1300 \mathrm{~V}$. $\mathbf{h}$, The jump in the measured DEA current indicates the time that the object makes contact with the DEA. Scale bars (e, g) are $5 \mathrm{~mm}$.

Despite having favorable properties such as robustness and self-sensing, DEAs present challenges for achieving flight due to their inherent nonlinearity. The strain in a DEA is proportional to the square of the applied electric field ${ }^{7}$. Consequently, a sinusoidal driving signal does not result in symmetric up stroke and down stroke motion (Fig. 3a and Supplementary Video 3) due to the influence of higher order harmonics (see Supplementary Information S2 for details on nonlinear actuation and higher harmonics). For example, when operated at $100 \mathrm{~Hz}$, the wing down stroke exhibits a slow reversal from $\mathrm{T}=0.5$ to $\mathrm{T}$ $=0.7$ (Fig. 3a and Supplementary Video 3). According to a previous aerodynamic study ${ }^{29}$, this slow wing 

147 lift force.

reversal can result in a substantial reduction in lift force. To mitigate the up stroke and down stroke asymmetry, we drive the DEA near the resonant frequency of the DEA-transmission-wing system to amplify the fundamental harmonic and attenuate higher harmonics. This asymmetry is substantially reduced when the DEA is driven at a frequency that is higher than half its resonance. Compared to flapping motion at $1 \mathrm{~Hz}$ or $100 \mathrm{~Hz}$, the slow wing reversal is negligible when the driving frequency increases to $280 \mathrm{~Hz}$ (Fig. 3b and Supplementary Video 3).

In addition to exhibiting nonlinear transduction, the DEA can undergo dynamic buckling that substantially affects flapping motion and reduces the lift force. When operated near the system resonance, the DEA experiences a large compressive load due to the drag force from the robot wing. This normal load causes the DEA to buckle along a direction perpendicular to its actuation axis. The actuator returns to its nominal configuration as this compressive load is reduced during wing reversal. In the next flapping period, the DEA buckles in the opposite direction due to the momentum of the restoring motion. Dynamic buckling substantially reduces the wing stroke amplitude (Fig. 3c-d and Supplementary Video 3), and it occurs at half the flapping frequency (Fig. 3d and Supplementary Video 3). Further, the large DEA deformation causes excessive electrode self-clearing and substantially reduces DEA performance and lifetime. Dynamic buckling can be inhibited by using circumferential constraints (in this case strings) to limit the DEA's off-axis motion at its mid-plane (Fig. 3e). Figure $3 \mathrm{f}$ shows the left-wing stroke amplitude as a function of driving frequency and voltage. The kinks of the green lines indicate stroke amplitude reduction due to dynamic buckling. Constraining the DEA's off-axis motion enables higher driving voltages and frequencies, which correspond to higher wing stroke amplitudes. The red shaded region indicates operating conditions that are inaccessible without constraining the DEA. Adding constraints increases the wing stroke peak-to-peak amplitude by approximately $25^{\circ}$, leading to a 1.6 times increase in 

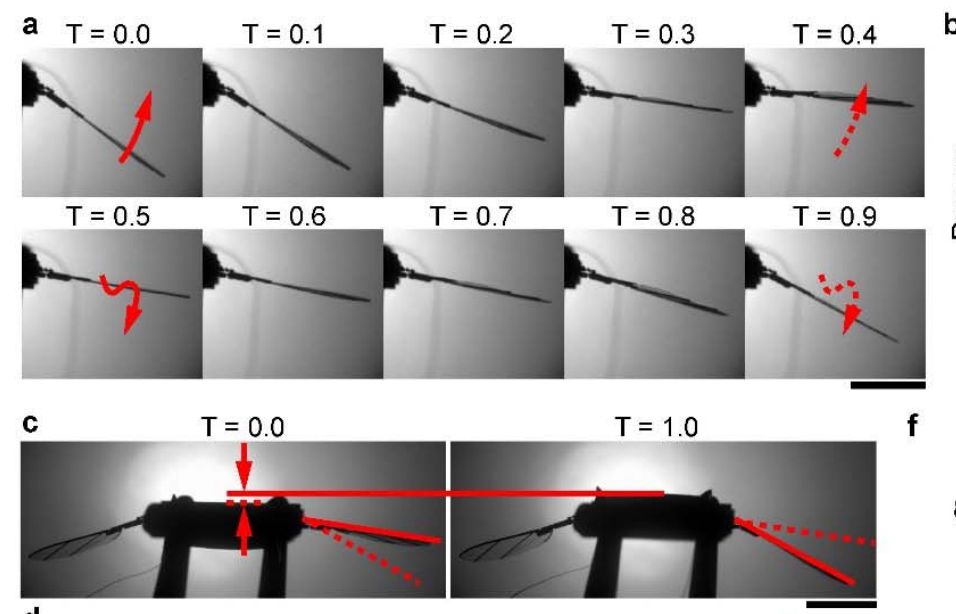

f

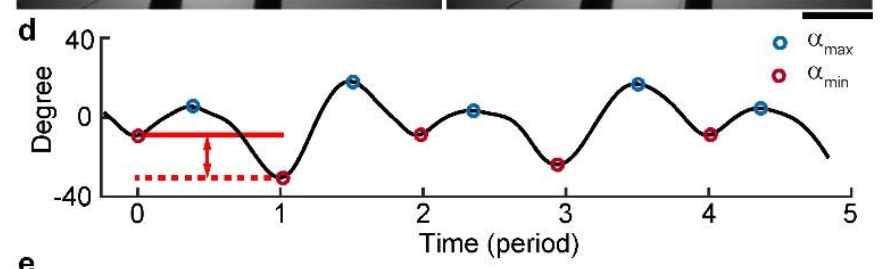

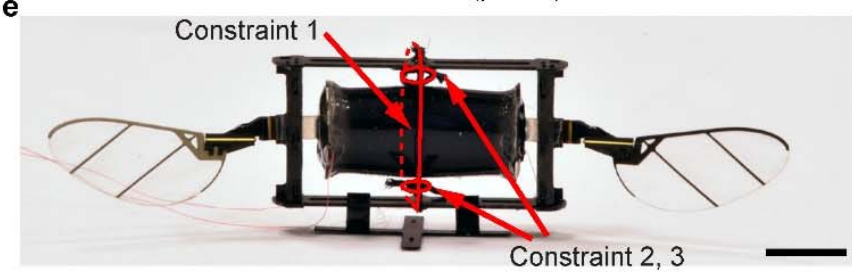
without constraining the DEA. Scale bars $(\mathbf{a}, \mathbf{c}, \mathbf{e})$ represent $5 \mathrm{~mm}$.
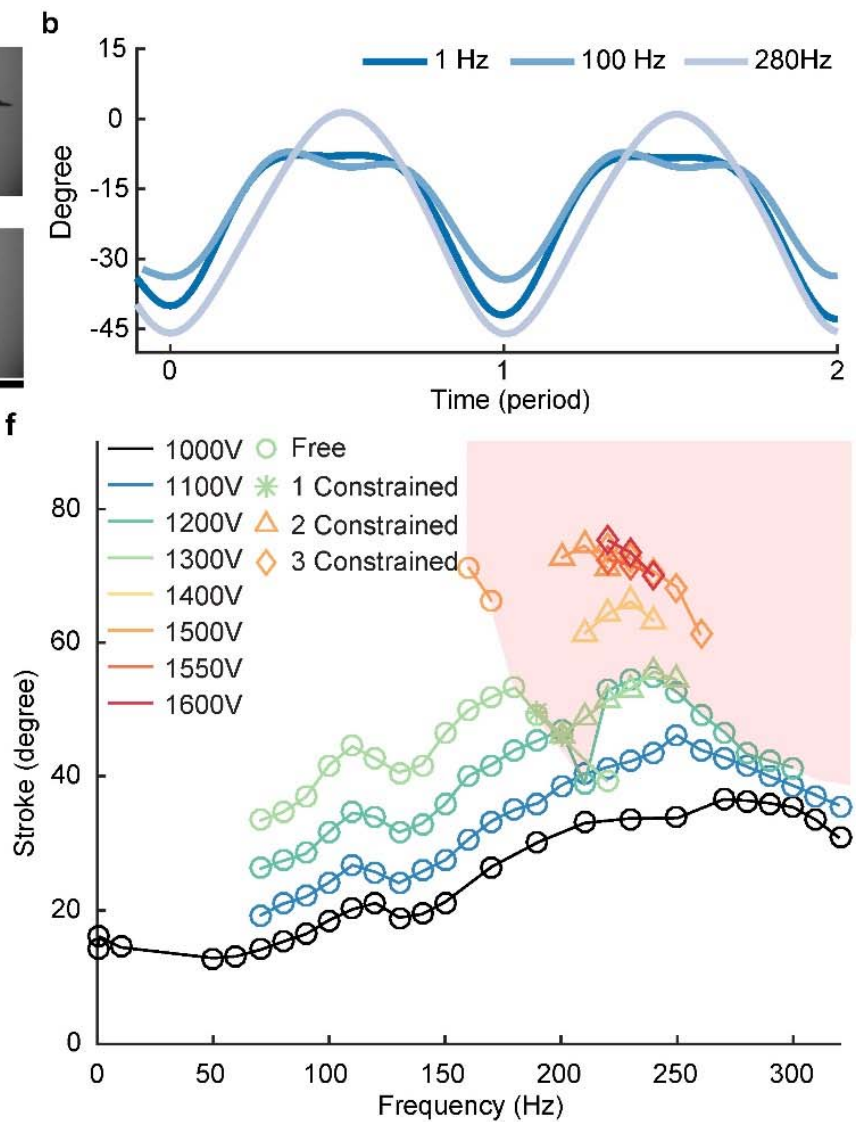

Figure 3 | DEA nonlinearity and dynamic buckling. a, Image sequence of the robot flapping motion at $100 \mathrm{~Hz}$ for one flapping period. The up stroke and down stroke are asymmetric. b, Tracked wing stroke motion at $1 \mathrm{~Hz}, 100 \mathrm{~Hz}$, and $280 \mathrm{~Hz}$. The wing stroke motion is asymmetric at low flapping frequencies. The nonlinear high frequency modes are reduced by postresonant inertial effects. c, Images that illustrate the DEA dynamic buckling. The red lines indicate that the DEA buckles and the wing stroke amplitude reduces. d, Tracked wing stroke motion that corresponds to the experiment shown in $\mathbf{c}$. The wing stroke amplitude reduces and the flapping period halves. e, Three pieces of thread circumferentially constrain the DEA to the robot airframe to eliminate out-of-plane motion and inhibit dynamic buckling. f, Stroke amplitude as a function of driving voltage and frequency. The shaded region (red) represents the stroke amplitudes and flapping frequencies that are unachievable

Addressing the challenges of nonlinear actuation enables flight demonstrations of the DEA-driven, flapping-wing microrobots. While all flight demonstrations are unconstrained, the robots carry a thin tether for offboard power supply and control. Driven by a single DEA, the $155 \mathrm{mg}$ robot demonstrates open-loop liftoff. The net lift generated by this MAV is approximately $1.8 \mathrm{mN}$, and it reaches a maximum height of $1.5 \mathrm{~cm}$ in $90 \mathrm{~ms}$ (Fig. 4a and Supplementary Video 4). To mitigate aerodynamic torque imbalances due to fabrication and assembly imprecision, a carbon fiber rod with a point mass is attached authority, this intrinsically unstable robot flips over within $110 \mathrm{~ms}$ of liftoff. 
To demonstrate stable ascending flight, we build a two actuator, four-winged robot (Fig. 1e) that utilizes precession around the body z-axis to achieve passive stability. We bias the resting wing pitch angle during robot assembly to induce a net yaw torque around the robot's body z-axis. The body z-component of the angular momentum induced by precession rejects the robot's pitch and roll torque imbalances. In an open loop takeoff experiment, we demonstrate that the robot reaches a height of $23.5 \mathrm{~cm}$ within 0.83 seconds of open-loop takeoff (Fig. 4b and Supplementary Video 5). We also construct a dynamical model and use numerical simulation to confirm the experimental observation on passive upright stability. Our simulation (Fig. 4c) shows the robot ascends $22.7 \mathrm{~cm}$ in 0.83 seconds with a yaw rate of $17.2 \mathrm{rev} / \mathrm{s}$. This passive stability property further enables us to operate more than one robot in a confined space without the need of motion tracking and feedback control. We demonstrate simultaneous takeoff flights of two robots (Supplementary Video 6) and show that they are robust against collisions with the surroundings and each other. In addition, passive stability and collision robustness can provide the ability to recover from inflight collisions or disturbances. Figure 4d and Supplementary Video 6 show a collision recovery flight in which the robot takes off from the center of a cylindrical shell, collides with the shell wall during its ascent, and continues to fly upward after making the collision. However, passive in-flight collision recovery is a probabilistic event that depends on the robot's flight speed and the collision impact. Without any robot attitude sensing and feedback control, the robot may be destabilized after experiencing one or multiple collisions (see Supplementary Information S3 for a detailed discussion on passive stability, collision recovery, and additional flight results).

To demonstrate controlled hovering flight, we design a four actuator, eight-winged robot (Fig. 1e) and use a motion tracking system ${ }^{3}$ and off-board computation for sensing and control (see Supplementary Information S4 for details on the controller design, implementation, experimental validation, and repeatability). Figure 4e shows composite images of a 16-second hovering flight, and the red dot indicates the desired setpoint. Figure $4 \mathrm{f}$ shows the corresponding trajectory of the same flight (Supplementary Video 
190 7), and the color scale represents the distance from the current position to the setpoint. For this 16 -second 191 flight, the maximum deviation of altitude, lateral position, and body angles are $12 \mathrm{~mm}(0.2$ body length 192 (BL)), $36 \mathrm{~mm}(0.6 \mathrm{BL})$, and $9^{\circ}$, respectively (Fig. 4g-i). 

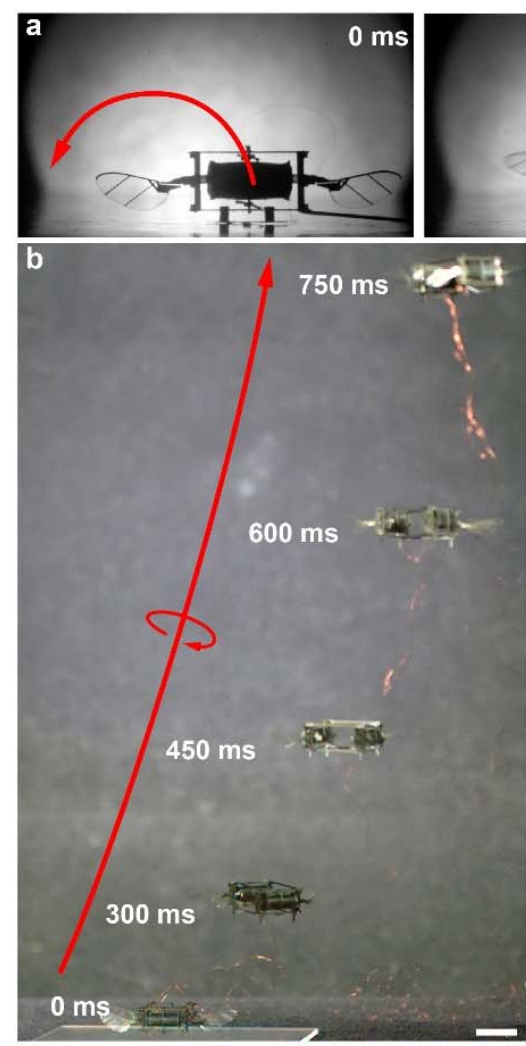

d

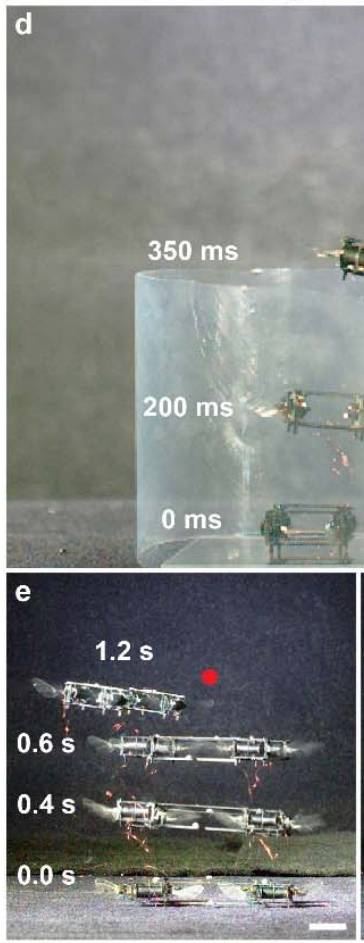

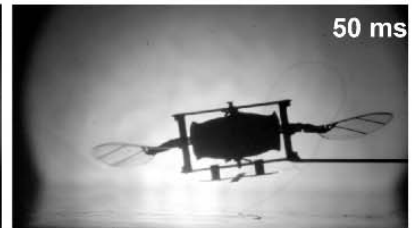

c

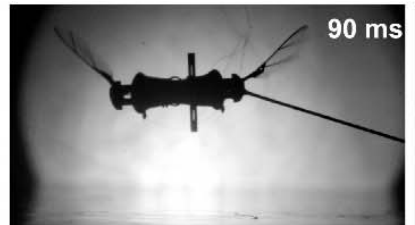

f
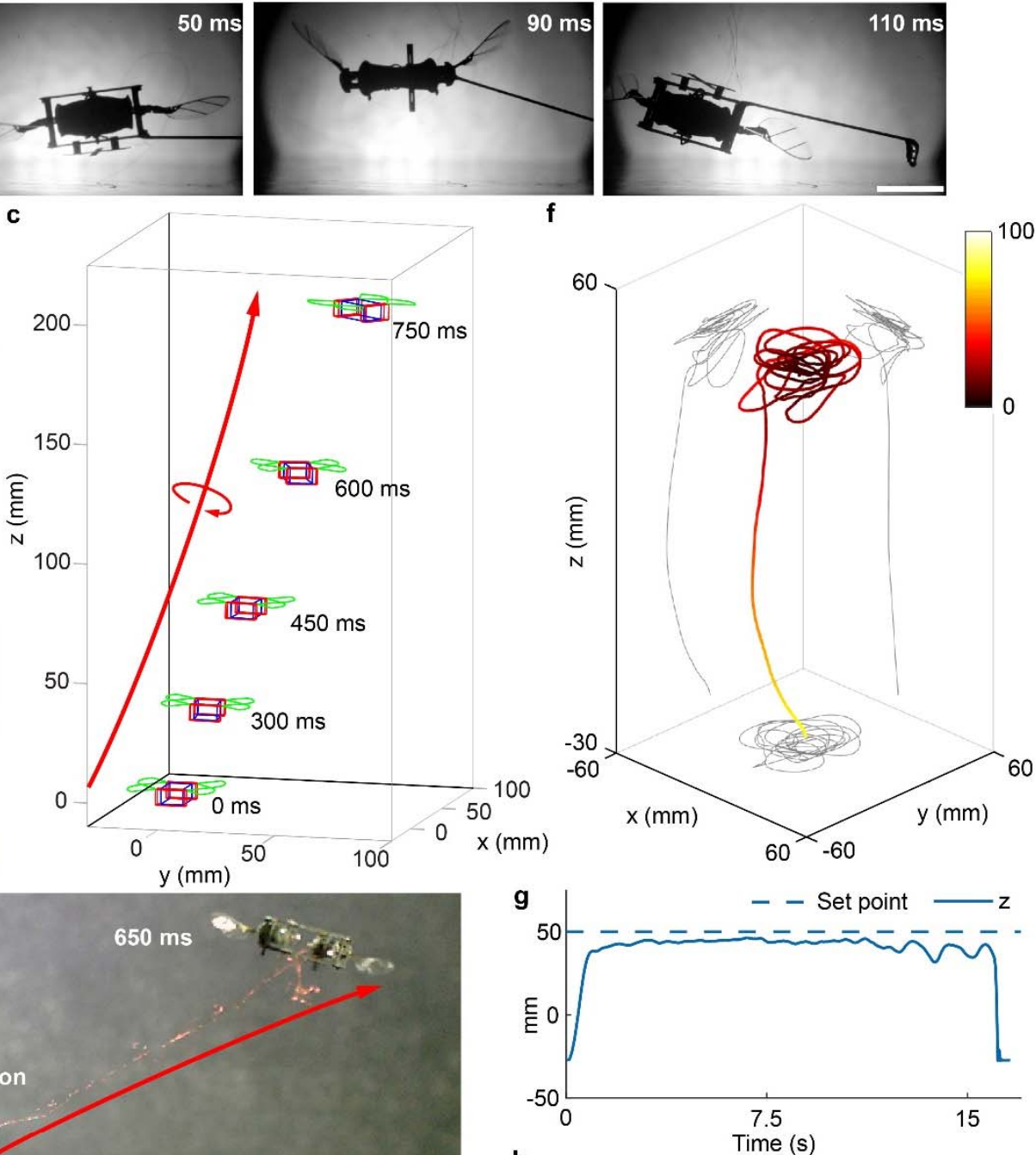

h

Time (s)
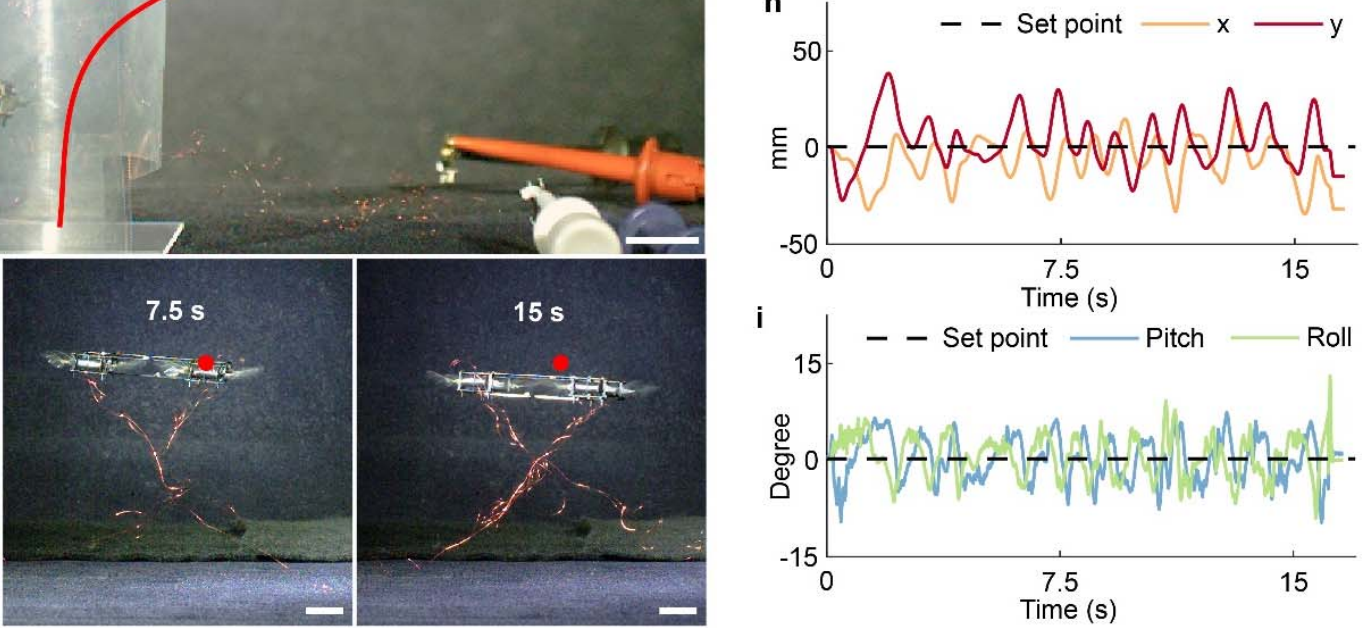

Time $(\mathrm{s})$

Figure 4 | Robot flight demonstrations. a, Unstable liftoff of a $155 \mathrm{mg}$ robot driven by one DEA. The robot reaches a height of $1.5 \mathrm{~cm}$ and then flips upside down due to unstable body pitch rotation. b, c, Passively stable ascending flight of a $320 \mathrm{mg}$ robot driven by two DEAs. Both the experiment (b) and the dynamical simulation (c) show the robot reaches approximately $23 \mathrm{~cm}$ within $0.75 \mathrm{~s}$ of open-loop takeoff. The simulation shows the robot precesses at a yaw rate of $17.2 \mathrm{rev} / \mathrm{s}$. d, The $320 \mathrm{mg}$ robot remains passively upright stable after colliding with an obstacle and continues to fly upward. e, Composite images of a 16-second controlled hovering flight that is demonstrated by a $660 \mathrm{mg}$ robot driven by four DEAs. f, The tracked flight trajectory corresponding to the experiment in $\mathbf{e}$. The color scale denotes the distance between the robot position and the set point. (g-i), Robot altitude (g), $\mathrm{x}$ and y positions (h), and attitude (i) as functions of time. Scale bars (a, b, d, e) are $1 \mathrm{~cm}$. 
To summarize, these flight demonstrations show the first time that soft artificial muscles have sufficient

204 power density to enable liftoff and have adequate bandwidth for flight control. Compared to the state-of205 the-art MAVs driven by microscale rigid actuators $(<500 \mathrm{mg})$, these soft actuator robots show advantages 206 such as in-flight robustness to collisions and self-sensing. A feature of the DEA's fabrication scalability 207 is that it enables efficient production of robotic modules that can be assembled in different configurations 208 for different functions. These properties will be important for enabling swarm flight of MAVs in highly 209 cluttered environments where collisions are difficult to avoid. However, compared to a recent 210 piezoelectric-actuator-driven $\mathrm{MAV}^{32}$ that can demonstrate power-autonomous takeoff flights, this robot consumes 15 times more input power and requires a drive voltage 6.5 times higher. The robot's weight and net lift are $170 \%$ and $75 \%$ that of the state-of-the-art piezoelectric-driven vehicle. To enable power autonomous flight in soft aerial robots, future studies need to reduce a soft actuator's operating voltage, improve its power efficiency, and further increase its power density. Reducing actuation voltage is crucial because up to $75 \%$ of the input electrical power can be dissipated by compact high-voltage boost and drive circuitry (as in a recent power autonomous $\mathrm{MAV}^{32}$ ). This challenge of lowering driving voltage can be tackled by refining DEA multi-layering techniques to further reduce the elastomer layer's thickness. Towards improving transduction efficiency, future studies can incorporate new architectures of electrically actuated soft actuators such as the electrohydraulic Peano-HASEL ${ }^{33}$ actuators that can use flexible metallic electrodes to reduce resistive losses. To increase power density, new electroactive polymers with higher dielectric strengths and lower viscoelasticity should be explored and incorporated into future soft artificial flight muscles. From a robot design perspective, scaling the vehicle size up can substantially mitigate the challenges associated with achieving power autonomy. A larger vehicle size can provide a larger net payload, which allows the robot to carry a larger and more efficient boost circuit. In addition, scaling up the wing size corresponds to a reduction of operating frequency, and leads to a linear increase in the DEA's power efficiency (see Methods section 3 and Supplementary Information S5 for a 227 detailed discussion on future directions to achieve power autonomous flights). More broadly and 
significantly, our work demonstrates that soft-actuated robots can be agile, robust, and controllable. These characteristics are important for developing future generations of soft robots for diverse applications such as environmental exploration and manipulation.

\section{References}

1 Dickerson, A.K., Shankles, P.G., Madhavan, N.M. \& Hu, D.L. Mosquitoes survive raindrop collisions by virtue of their low mass. Proceedings of the National Academy of Sciences 109, 98229827 (2012).

2 Mountcastle, A. M. \& Combes, S. A. Biomechanical strategies for mitigating collision damage in insect wings: structural design versus embedded elastic materials. Journal of Experimental Biology 217, 1108-1115 (2014).

3 Ma, K. Y., Chirarattananon, P., Fuller, S. B. \& Wood, R. J. Controlled flight of a biologically inspired, insect-scale robot. Science 340, 603-607 (2013).

4 Chen, Y. et al. A biologically inspired, flapping-wing, hybrid aerial-aquatic microrobot. Science Robotics 2, eaao5619 (2017).

5 Zou, Y., Zhang, W. \& Zhang, Z. Liftoff of an electromagnetically driven insect-inspired flappingwing robot. IEEE Transactions on Robotics 32, 1285-1289 (2016).

6 De Croon, G., De Clercq, K., Ruijsink, R., Remes, B. \& De Wagter, C. Design, aerodynamics, and vision-based control of the DelFly. International Journal of Micro Air Vehicles 1, 71-97 (2009).

7 Carpi, F., Bauer, S., \& Rossi, D. D. Stretching dielectric elastomer performance. Science 330, 1759-1761 (2010).

8 Rosset, S. \& Shea, H. R. Flexible and stretchable electrodes for dielectric elastomer actuators. Applied Physics A 110, 281-307 (2013).

9 Zhao, H. et al. Compact dielectric elastomer linear actuators. Advanced Functional Materials 28, 1804328 (2018).

10 Acome, E., et al. Hydraulically amplified self-healing electrostatic actuators with muscle-like performance. Science 359, 61-65 (2018).

11 Pelrine, R. et al. Dielectric elastomer artificial muscle actuators: toward biomimetic motion. in Smart Structures and Materials 2002: Electroactive polymer actuators and devices (EAPAD). 126-138 (2002).

12 Zhao, J., Niu, J., McCoul, D., Leng, J. \& Pei, Q. A rotary joint for a flapping wing actuated by dielectric elastomers: design and experiment. Meccanica 50, 2815-2824 (2015).

13 Lau, G.-K., Lim, H.-T., Teo, J.-Y. \& Chin, Y.-W. Lightweight mechanical amplifiers for rolled dielectric elastomer actuators and their integration with bio-inspired wing flappers. Smart Materials and Structures 23, 025021 (2014).

14 Shepherd, R. F. et al. Multigait soft robot. Proceedings of the national academy of sciences $\mathbf{1 0 8}$, 20400-20403 (2011).

15 Rus, D. \& Tolley, M. T. Design, fabrication and control of soft robots. Nature 521, 467 (2015).

16 Majidi, C. Soft robotics: a perspective - current trends and prospects for the future. Soft Robotics 1, 5-11 (2014). 
17 Choe, K. et al. Performance Characteristics of Electro-chemically Driven Polyacrylonitrile Fiber Bundle Actuators. Journal of intelligent material systems and structures 17, 563-576 (2006).

18 Suzumori, K., Iikura, S. \& Tanaka, H. Applying a flexible microactuator to robotic mechanisms. IEEE Control systems magazine 12, 21-27 (1992).

19 Onal, C. D., Chen, X., Whitesides, G. M. \& Rus, D. Soft mobile robots with on-board chemical pressure generation. in Robotics Research 525-540 (2017).

20 Katzschmann, R. K., Marchese, A. D. \& Rus, D. Hydraulic autonomous soft robotic fish for 3D swimming. in Experimental Robotics. 405-420 (2016).

21 Loomis, J., Xu, P. \& Panchapakesan, B. Stimuli-responsive transformation in carbon nanotube/expanding microsphere-polymer composites. Nanotechnology 24, 185703 (2013).

22 Cheng, N. G., Gopinath, A., Wang, L., Iagnemma, K. \& Hosoi, A. E. Thermally tunable, selfhealing composites for soft robotic applications. Macromolecular Materials and Engineering 299, 1279-1284 (2014).

23 Sadeghipour, K., Salomon, R. \& Neogi, S. Development of a novel electrochemically active membrane and'smart'material based vibration sensor/damper. Smart Materials and Structures 1, 172 (1992).

24 Pei, Q., Rosenthal, M., Stanford, S., Prahlad, H. \& Pelrine, R. Multiple-degrees-of-freedom electroelastomer roll actuators. Smart materials and structures 13, N86 (2004).

25 Shian, S., Bertoldi, K. \& Clarke, D. R. Use of aligned fibers to enhance the performance of dielectric elastomer inchworm robots. in Electroactive Polymer Actuators and Devices (EAPAD). 94301P (2015).

$26 \mathrm{Li}, \mathrm{T}$. et al. Fast-moving soft electronic fish. Science Advances 3, e1602045 (2017).

27 Berlinger, F. et al. A Modular Dielectric Elastomer Actuator to Drive Miniature Autonomous Underwater Vehicles. in IEEE International Conference on Robotics and Automation (ICRA). 3429-3435 (2018).

28 Dickinson, M. H. Muscle efficiency and elastic storage in the flight motor of Drosophila. Science 268, 87-90 (1995).

29 Chen, Y., Gravish, N., Desbiens, A. L., Malka, R. \& Wood, R. J. Experimental and computational studies of the aerodynamic performance of a flapping and passively rotating insect wing. Journal of Fluid Mechanics 791, 1-33 (2016).

30 Tu. Z., Fei. F., Zhang. J. \& Deng. X. Acting is Seeing: Navigating Tight Space Using Flapping Wings. in IEEE International Conference on Robotics and Automation (ICRA) (2019).

31 Jayaram, K., Jafferis, N. T., Doshi, N., Goldberg, B. \& Wood, R. J. Concomitant sensing and actuation for piezoelectric microrobots. Smart Materials and Structures 27, 065028 (2018).

32 Jafferis, N.T., Helbling, E.F., Karpelson, M. \& Wood, R.J. Untethered flight of an insect-sized flapping-wing microscale aerial vehicle. Nature 570, 491-495 (2019)

33 Kellaris, N., Venkata, V. G., Smith, G. M., Mitchell, S. K., \& Keplinger, C. Peano-HASEL actuators: Muscle-mimetic, electrohydraulic transducers that linearly contract on activation. Science Robotics, 3(14), eaar3276.

\section{Acknowledgement}

We thank Yishan Zhu for comments and discussions. This work is supported by the National Science Foundation (award \#CMMI-1830291) and the Wyss Institute for Biologically Inspired Engineering. In addition, the prototypes were enabled by equipment supported by the Army Research Office DURIP program (award no. W911NF-13-1-0311). Any opinions, findings, and conclusions or recommendations expressed in this material are those of the authors and do not necessarily reflect the views of the National

315 Science Foundation. 


\section{Author Contribution}

317 Y.C., H.Z., and R.J.W proposed and designed the research; Y.C., H.Z., and J.M. built the robot; Y.C., 318 H.Z., J.M., P.C., and E.H. conducted the experimental work; Y.C., H.Z., P.C., N.P.H., D.C., and R.J.W 319 contributed to modelling and data analysis; Y.C. wrote the paper. All authors provided feedback.

\section{Data Availability}

321 All data generated or analyzed for this paper are included in the published article, its Methods, and 322 Supplementary Information. Original videos, computer code, and sensor data are available from the 323 corresponding author on reasonable request. 
325 Methods

326 1. Conceptual design of a DEA-powered aerial robot

327 The DEA-powered robot consists of five major components: an actuator, an airframe, transmission, two 328 wing hinges, and two wings. The two ends of the DEA are connected to the robot transmission, and the 329 DEA's linear actuation is converted to the flapping motion of both wings. The structural design of this 330 robot is similar to that of a microrobot powered by piezoelectric actuators presented in a previous study ${ }^{34}$. 331 However, we need to redesign each component to accommodate the soft actuator. In the following, we 332 describe the design process to determine key robot parameters and present the requirements on DEA 333 performance.

To achieve takeoff, the DEA must satisfy requirements for blocked force, resonant frequency, free displacement, and power density. Specifically, the actuator needs to meet two conditions. First, the robot wings need to flap at sufficient frequency with adequate amplitude to generate a lift force that balances the robot weight. This condition places requirements on the DEA's operating frequency and displacement. Rearranging the equation that imposes the lift force and robot weight balance leads to the relationship:

where $f$ is the robot's operating frequency, $\delta$ is half of the DEA's free displacement at the frequency $f$, $341 \hat{r}_{2}$ is the wing's second area moment, $R$ is the wing span, $T$ is the transmission ratio, $A R$ is the wing's 342 aspect ratio, $W$ is the robot weight, $\overline{C_{L}}$ is the mean lift coefficient, $\rho$ is the air density, and $f_{m}$ is a scaling ratio such that the extra lift force can be used for flight control. In addition to satisfying this kinematic condition, the DEA needs to overcome the aerodynamic drag force during flight, and this imposes a requirement on the DEA's blocked force:

$$
F_{B}=2 \sqrt{2} T r_{c p} W f_{m} \frac{\overline{C_{D}}}{\overline{\bar{C}_{L}}}
$$


where $F_{B}$ is the DEA's blocked force, $r_{c p}$ is the wing's spanwise center of pressure, and $\overline{C_{D}}$ is the time averaged drag coefficient. The derivation of equations (1) and (2) closely follows from equations (1-14) in a previous work ${ }^{34}$. In equations (1) and (2), we assume that the DEA's blocked force is independent of its actuation frequency. This assumption is validated in the next section on DEA characterization. Multiplying equations (1) and (2) gives a requirement for the DEA's output mechanical power.

The design of a DEA-powered aerial robot also needs to satisfy an additional condition because the DEA's actuation is nonlinear with respect to input voltage. With a sinusoidal input, the DEA's actuation contains higher order harmonics that can adversely affect flapping wing kinematics. As discussed in the main text, we attenuate higher order harmonics by setting the robot operating frequency close to the natural frequency of the DEA-transmission-wing system. A previous study ${ }^{34}$ shows the actuator-transmissionwing system can be described by a lumped-parameter model. The system resonant frequency is given by:

$$
f=2 \pi \sqrt{\frac{k_{m}+k_{h} T^{2}}{m_{a}+2 T^{2} I_{z z}}},
$$

where $k_{m}$ is the DEA's intrinsic stiffness, $m_{a}$ is the DEA mass, $k_{h}$ is the transmission's torsional stiffness, and $I_{z z}$ is the wing's moment of inertia relative to the stroke rotational axis. For our robot, the transmission stiffness is much lower than the DEA's effective stiffness. To obtain a higher operating frequency, this condition requires a smaller wing moment of inertia. The wing moment of inertia can be decreased by reducing wing size. Using equations (1) - (3), we select values for the transmission ratio and the wing size while satisfying constraints imposed by our fabrication methods (i.e., minimum feature size, wing inertia, etc). The values of these design parameters are reported in Extended Data Table 1. Using these parameters, we obtain the following requirements for a $100 \mathrm{mg}$ DEA: $F_{B}=0.2 \mathrm{~N}, f=290 \mathrm{~Hz}$, and $\delta=0.3 \mathrm{~mm}$. Multiplying these parameters shows that the DEA needs to have a minimum output power density of $200 \mathrm{~W} / \mathrm{kg}$. This requirement is similar to that of the MAVs powered by piezoelectric actuators ${ }^{3}$ and to the power density values estimated for flying insects. 


\section{Fabrication of robot components}

The robot airframe, transmission, wings, and wing hinges are made using an existing multi-scale, multimaterial fabrication method ${ }^{35}$. The airframe consists of eight pieces of $160 \mu \mathrm{m}$ carbon fiber laminates assembled manually and reinforced with Loctite 495 (Extended Data Figure 1a). The robot transmission is a planar four-bar mechanism. The transmission ratio is approximated as $T=l_{3}^{-1}$, where the link length $l_{3}$ is marked in Extended Data Figure 1b. The robot transmission is attached to the DEA via a fiber glass connector, which insulates the robot structure from the DEA's driving signals. Further, the transmission connects the airframe and the wing hinge. A wing is attached onto the robot's wing hinge. The wing hinge and wing are designed based on an existing method ${ }^{36}$, and their geometries are illustrated in Extended Data Figure 1c and d.

The DEA takes the form of a cylindrical shell, whose height and radius determine the actuation frequency, blocked force, and free displacement. The DEA is made of a multi-layering process ${ }^{9}$, and it is rolled from a rectangular elastomer sheet that has embedded electrodes. Since the DEA drives two wings simultaneously, its free displacement needs to be larger than $600 \mu \mathrm{m}$ (twice the value of the design parameter $\delta$ ). Based on the values of DEA free displacement, peak loading, and elastomer stiffness, we set the actuator length to $8 \mathrm{~mm}$. To obtain a blocked force over $0.2 \mathrm{~N}$, the elastomer sheet (prior to roll up) width is set to $5 \mathrm{~cm}$. This elastomer sheet is approximately $220 \mu \mathrm{m}$ thick, and it is manually rolled into a cylindrical shell whose inner and outer diameters are $1.5 \mathrm{~mm}$ and $4.5 \mathrm{~mm}$, respectively.

The elastomer is a 5:4 mixture of Ecoflex 0030 (Smooth-On) and Sylgard 184 (Dow Corning). The ratio of crosslinker in Sylgard 184 is 1:40. We put a thin layer of CNT (from Nano-C Inc, Westwood, MA) on the elastomer and use it as the DEA's compliant electrode. For coating the electrode, we use $150 \mu \mathrm{L}$ of CNT solution over a $90 \mathrm{~mm}$ diameter PTFE filter (Satorius 7022P). The procedures for elastomer preparation, spin coating, and electrode patterning are adopted from a previous study ${ }^{9}$. 
We made several modifications to the fabrication process to increase DEA power density and endurance.

394 First, DEA power density can be increased by having an even number of CNT layers. Extended Data 395 Figure 1e shows the rolling process of a multi-layered DEA. We use grey colored regions to denote the elastomer layers. The positive and negative electrodes are represented by red and black lines, respectively. We represent the bottom elastomer layer with a darker grey color. When the elastomer sheet is rolled into a cylindrical shell, the DEA's bottom layer is put into contact with its top layer. This is illustrated by the inset shown in Extended Data Figure 1f. The region highlighted by blue lines further shows that a new layer is formed by the DEA's top and bottom elastomer layers and electrodes. If the top and bottom electrodes are oppositely charged (as illustrated in Extended Data Figure 1f), then this effective layer 402 403 develops an electric field and contributes to actuation. We must have an even number of electrode layers to ensure the bottom and top electrodes are oppositely charged. In this work, our DEA design has six CNT and seven elastomer layers. Further, if the top and bottom elastomer layers have the same thickness as all other layers, then the electric field in this new layer is only half that of other layers because the effective 406 layer thickness is $t_{\text {top }}+t_{\text {bottom }}$ (Extended Data Figure 1g). Hence, reducing the top and bottom layer thickness increases the electric field in the additional layer, and this results in an increase in DEA output power. We use a faster spin coating speed (2700 rpm) for the top and bottom layer and slower speed (1700 rpm) for the middle layers. Through reducing the top and bottom elastomer layer thickness by approximately 35\% (Extended Data Figure 1h), we obtain an 11\% mass reduction and a 9\% increase in output power relative to a DEA with constant elastomer layer thickness. After making the elastomer layers and transferring the electrodes, we cut out the DEA from the elastomer substrate and roll it into a cylindrical shell. In the previous study ${ }^{9}$, the DEA is cut out manually with a razor blade. Our application requires higher accuracy, so we program a digital cutter (Silhouette Cameo) to cut out the DEA. The DEA's length is set to $8.6 \mathrm{~mm}$ including the exposed CNT tabs for electrical connection. With this modification, variation in the DEA length is reduced to within $150 \mu \mathrm{m}$. Having a precise DEA length is 417 crucial for attaching the DEA to the robot transmission during assembly. 
In addition, the DEA's bandwidth depends on several factors such as elastomer mechanical

419 viscoelasticity $(\tan \delta)$, DEA geometry, and electrode conductivity. Here, we improve the fabrication 420 process relative to a previous study ${ }^{9}$ to ensure good conductivity during DEA actuation (Extended Data 421 Figure 1i). After the DEA is rolled into a shell, carbon conductive adhesive (Electron Microscopy 422 Sciences) is applied to the exposed electrodes and carbon fiber endcaps are glued to each end. For driving 423 our flapping wing robot, the DEA needs to overcome aerodynamic drag during both elongation and 424 retraction phases. During DEA retraction, aerodynamic drag opposes the DEA motion and applies a tensile 425 stress on the DEA connections. At peak loading, this tensile stress weakens the bonding between the 426 elastomer and the endcap, and it can create local tears and further lead to delamination. This delamination 427 reduces electrical conductivity, which increases the DEA's time constant and reduces its bandwidth. We 428 overcome this problem by modifying the fabrication process to increase the end cap adhesion strength. 429 During fabrication, Loctite 416 is applied to the outer perimeter of the elastomer shell and the endcaps. The DEA is compressed with a mass of $18 \mathrm{~g}$ and then baked at $72{ }^{\circ} \mathrm{C}$ for 4 hours. The glue cures in this process and holds the electrical connections in compression. The preload is removed after the glue cures, and other regions of the DEA return to a neutral state. A photograph of the DEA is shown in Extended Data Figure 1j. With this procedure, we obtain an increase in DEA conductivity of approximately four times compared to those made using previous methods ${ }^{9}$.

\section{DEA performance characterization}

Here we describe the experimental characterization of the DEA's blocked force, free displacement, bandwidth, power consumption, and efficiency. To measure the DEA's blocked force, we place the DEA under a force sensor (Nano 17 Titanium). The sensor is mounted on a two-axis stage and is lowered until it touches the DEA's top cap (Extended Data Figure 2a). To ensure the DEA remains securely affixed under the sensor during its retraction phase, we continue lowering the sensor to induce a preload of approximately $0.05 \mathrm{~N}$. The sensor resolution and the resonant frequency are $1.5 \mathrm{mN}$ and $3000 \mathrm{~Hz}$, 
post-process the data. To measure the DEA's free displacement, we place a DEA under a laser vibrometer

444 (Polytec PSV-500). The vibrometer measures the instantaneous velocity of the DEA's oscillatory motion 445 (Extended Data Figure 2b) approximately 40 times per period. For time sequence measurements, the 446 vibrometer averages over five cycles to reduce measurement noise. The measured velocity is integrated numerically to calculate the DEA displacement. In addition, the vibrometer can measure the DEA's frequency response by driving the DEA with white noise and computing the Fast Fourier Transform (FFT) of the displacement. This measurement gives a linear approximation of the device frequency response. It quantifies the DEA's resonant modes and phase shift (Figure 2c-d). This information is useful for robot design because the DEA's motion is approximated as linear around system resonance at flight conditions.

Sample experimental measurements of blocked force and free displacement are shown in Extended Data Figure 2c and d, respectively. In these experiments, the DEA is driven at $350 \mathrm{~Hz}$ and $1300 \mathrm{~V}$. The amplitude of the DEA's blocked force is calculated as the maximum value of the measured force and it does not include the preload force (the range is labelled by the red arrows in Extended Data Figure 2c). In our experiments, we vary the preload in the range of $0.025 \mathrm{~N}$ to $0.1 \mathrm{~N}$ and find that the magnitude of 457 preload has a negligible effect on the blocked force measurement. The amplitude of the DEA's free 458 displacement is calculated as the difference between the maximum and the minimum value (as indicated 459 by the red arrows in Extended Data Figure 2d). We report the peak-to-peak displacement value because 460 the DEA does mechanical work during both elongation and retraction. To characterize DEA performance 461 for different operating conditions (Figure 2a-b), we vary input voltage amplitudes and driving frequencies 462 from $800 \mathrm{~V}$ to $1300 \mathrm{~V}$, and from $1 \mathrm{~Hz}$ to $600 \mathrm{~Hz}$. Based on the force and displacement measurements, the 463 actuator energy and power density are calculated as:

$$
e=\frac{1}{2 m_{a}} F_{B} \delta
$$

$$
p=\frac{1}{2 m_{a}} F_{B} \delta f
$$


466 Equations 4 and 5 assume the elastomer's stress-strain relationship is approximately linear. Through 467 conducting tensile tests using an Instron materials testing machine, we find the elastomer exhibits a linear 468 response for a strain less than $20 \%$. The elastomer Young's modulus is measured to be $140 \mathrm{kPa}$. The 469 maximum measured energy density (Extended Data Figure 2e) and power density (Extended Data Figure $4702 \mathrm{f}$ ) are $1.13 \mathrm{~J} / \mathrm{kg}$ and $563 \mathrm{~W} / \mathrm{kg}$, respectively (at $500 \mathrm{~Hz}, 1300 \mathrm{~V}$ ). These values satisfy the criteria for robot takeoff (Supplementary Information S1). The DEA's driving voltage can be further increased to $4721500 \mathrm{~V}$ in controlled hovering flight demonstrations, so the DEA's peak power density is estimated to be $47315 \%$ higher than the reported value. The DEA experiences dielectric breakdown for a driving voltage 474 higher than $1500 \mathrm{~V}$.

475 In our flight experiments, the robot is driven by an external power source through a thin tether. Here we 476 quantify the DEA's resistance, capacitance, power consumption, and efficiency. These parameters are 477 important for achieving power autonomous flights in future studies. To quantify the DEA's power 478 consumption, we measure the DEA's input voltage $(V)$ and corresponding current $(I)$ at flight conditions. 479 The average electrical power input is:

$$
\bar{P}_{\text {in }}=\frac{1}{T} \int_{0}^{T} V(t) I(t) d t
$$

A sample measurement of instantaneous power is shown in Extended Data Figure 2g, in which the average power consumption is $450 \mathrm{~mW}$. We further measure the DEA's resistance and capacitance by sending a step input and measuring the corresponding current response. The system is modelled as a RC circuit, and parameters such as series resistance, capacitance, and time constant can be obtained by fitting a first order system to the current response. The DEA's resistance, capacitance, and time constant are $170 \mathrm{k} \Omega, 1.04$ $\mathrm{nF}$, and $178 \mu \mathrm{s}$, respectively. Having calculated the DEA's resistance, we further compute the power 487 dissipated due to electrical resistance:

$$
\bar{P}_{\text {ele }}=\frac{R}{T} \int_{0}^{T} I^{2}(t) d t
$$


The DEA electrode dissipates $330 \mathrm{~mW}$ of power at flight conditions. The mechanical power output at this operating condition is calculated as $P=\frac{1}{2} F_{B} \delta f$, where the values of $F_{B}, \delta$, and $f$ are $0.19 \mathrm{~N}, 0.89 \mathrm{~mm}$, and $300 \mathrm{~Hz}$, respectively. The estimated power output is $25 \mathrm{~mW}$, which implies the DEA efficiency is $5.6 \%$. Over $73 \%$ of the power is dissipated by the electrode resistance, and the rest of the power dissipation is contributed by the elastomer's viscoelastic damping.

This power dissipation leads to substantial heating of the DEA. The system can be described by a first order conduction model:

$$
\frac{d T}{d t}=-K\left(T-T_{a}\right)+\frac{Q}{C^{\prime}}
$$

where $T$ is the DEA temperature, $T_{a}$ is the ambient temperature, $K$ is the dissipation rate, $Q$ is the heat inflow, and $C$ is the DEA's heat capacity. This first order differential equation has a closed form solution. The solutions for the rising and the cooling phases are:

$$
\begin{aligned}
& T_{\text {rise }}=T_{a}+\frac{Q}{c K_{1}}\left(1-e^{-K_{1} t}\right), \\
& T_{\text {cool }}=T_{a}+\left(T_{i}-T_{a}\right) e^{-K_{2} t},
\end{aligned}
$$

where $T_{i}$ is the initial temperature at the onset of cool down. The dissipation coefficients $\left(K_{1}\right.$ and $\left.K_{2}\right)$ in the heating and the cooling phases are different because the flapping motion during the heating phase induces an airflow that facilitates convective cooling. The values of these modeling parameters are reported in Extended Data Table 1.

We use a FLIR T440 thermal camera to measure the DEA temperature when the robot operates under takeoff conditions (Extended Data Figure 2h). The DEA temperature increases from $28{ }^{\circ} \mathrm{C}$ to $70{ }^{\circ} \mathrm{C}$ in 90 seconds. An analytical fit is superimposed on the same graph (Extended Data Figure 2h). Snapshots of a thermal video are shown in Extended Data Figure 2i. The maximum DEA temperature reaches $70.0{ }^{\circ} \mathrm{C}$ before cool down. This experiment shows most of the input electrical power is dissipated in the form of 
511 heat. Generating excessive amount of heat can lead to thermal failure and reduce actuator lifetime. 512 Through our experiments, we find our DEA can operate for over 600,000 cycles under takeoff conditions, 513 equivalent to 33 minutes of flight time.

514 In this study, our DEA has a low transduction efficiency of 5.6\%. This low transduction efficiency would 515 not be conducive to power autonomous flights. In addition, it requires a $1300 \mathrm{~V}$ driving signal to achieve 516 takeoff, which creates challenges for developing high efficiency boost circuitry. While this study does not 517 aim to achieve power autonomous flight, it is important to identify major challenges and potential 518 solutions. Future studies should focus on increasing the DEA electrode's conductivity, reducing elastomer 519 layer thickness to reduce the driving voltage, and redesigning the DEA geometry and robot wings to 520 reduce the flapping frequency. First, increasing electrode conductivity will lead to a reduction of resistive 521 power loss. This can be done by exploring new electrode materials such as a hybrid network of carbon 522 nanotubes, graphene and silver nanowires ${ }^{37}$ or intrinsically stretchable electrodes such as conductive 523 hydrogels ${ }^{38}$ or liquid metal. Second, reducing elastomer thickness will reduce the operating voltage. We 524 can achieve this by increasing the spin coating speed or exploring alternative method such as using an 525 automatic thin film applicator. Further, the spin coating and the electrode transfer process can be done in 526 a clean room environment to reduce the number of particulates in the elastomer and on the electrodes. 527 Third, new electroactive materials such as bottlebrush elastomers ${ }^{39}$ can be explored to further increase the 528 actuator's energy density. In addition, our experiments show that DEA power consumption is linearly 529 proportional to its operating frequency. To reduce power expenditure, future studies can redesign the DEA 530 geometry and robot transmission to reduce system resonant frequency. Alternatively, nonlinear controllers 531 can be developed so that the DEA motion does not need to be linearized around its resonance. Beyond 532 improving the DEAs, we can apply a new class of electrostatic actuators named Peano-HASEL ${ }^{33,40}$ that $^{-H^{2}}$ 533 have shown promise for achieving very high energy density and moderate bandwidth. For that class of 534 actuators, it would be important to work on device miniaturization to reduce the driving voltage. 
536 Methods Reference

537 34. Whitney, J.P., \& Wood, R. J. Conceptual design of flapping-wing micro air vehicles. Bioinspiration 538 \& Biomimetics. 7(3), 036001 (2012).

539 35. Whitney, J. P., Sreetharan, P. S., Ma, K. Y., \& Wood, R. J. Pop-up book MEMS. Journal of 540 Micromechanics and Microengineering. 21, 115021 (2011).

541 36. Chen, Y., Ma, K., \& Wood, R. J. Influence of wing morphological and inertial parameters on flapping 542 flight performance. In IEEE/RSJ International Conference on Intelligent Robots and Systems. 2329-2336 543 (2016).

544 37. Liang, J., Li, L., Chen, D., Hajagos, T., Ren, Z., Chou, S. Y., Hu, W., \& Pei, Q. Intrinsically stretchable 545 and transparent thin-film transistors based on printable silver nanowires, carbon nanotubes and an 546 elastomeric dielectric. Nature communications, 6, 7647 (2015).

547 38. Keplinger, C., Sun, J. Y., Foo, C. C., Rothemund, P., Whitesides, G. M., \& Suo, Z. Stretchable, 548 transparent, ionic conductors. Science, 341(6149), 984-987 (2013).

549 39. Vatankhah-Varnoosfaderani, M., Daniel, W. F., Zhushma, A. P., Li, Q., Morgan, B. J., Matyjaszewski, 550 K., et al. Bottlebrush elastomers: A new platform for freestanding electroactuation. Advanced Materials, 551 29(2), 1604209 (2017).

552 40. Kellaris, N., Venkata, V. G., Rothemund, P., \& Keplinger, C. An analytical model for the design of 553 Peano-HASEL actuators with drastically improved performance. Extreme Mechanics Letters, 100449 554 (2019). 
Extended Data Figures and Tables

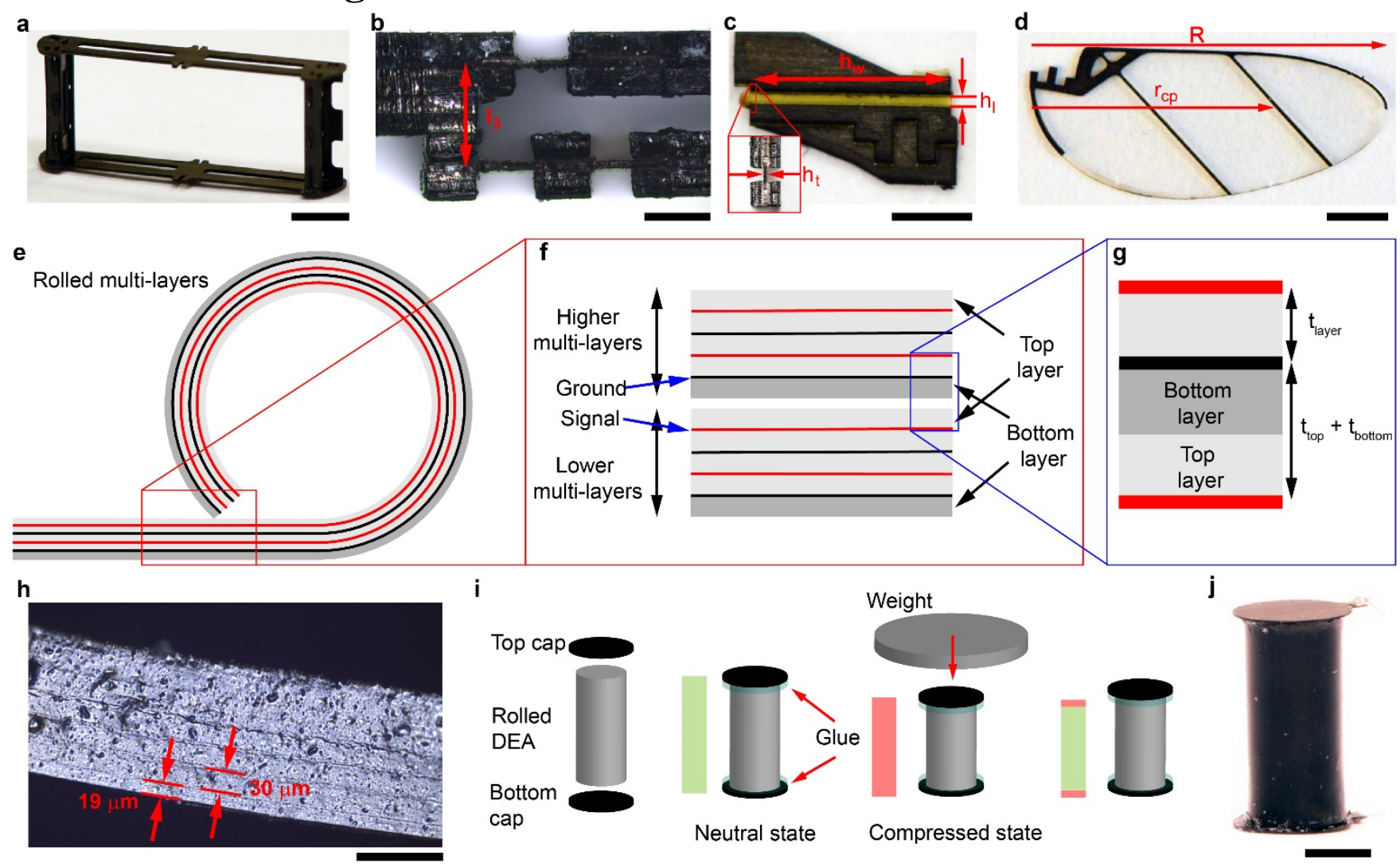

Extended Data Figure 1 | Design and fabrication of robot components. a, A $40 \mathrm{mg}$ airframe made of eight pieces of carbon fiber composites. Scale bar represents $2 \mathrm{~mm}$. b, Top view of the planar four-bar transmission. The red arrow marks $l_{3}$, which is the inverse of the transmission ratio T. Scale bar represents $200 \mu \mathrm{m}$. c, Front view of the wing hinge. The hinge width $\left(h_{w}\right)$, length $\left(h_{l}\right)$, and thickness $\left(h_{t}\right)$ determine its torsional stiffness. Scale bar represents $1 \mathrm{~mm}$. d, Front view of a robot wing whose wing span $(R)$ and center of pressure $\left(r_{c p}\right)$ are $10 \mathrm{~mm}$ and $7 \mathrm{~mm}$, respectively. Scale bar represents $2 \mathrm{~mm}$. e, An illustration of rolling an elastomer-electrode multilayer into a DEA. f, A zoomed-in illustration of the inset shown in e. $\mathbf{g}$, A zoomed-in illustration of the inset shown in $\mathbf{f}$. The bottom layer of the top multi-layer and the top layer of the bottom multi-layer forms a region that can be actuated. $\mathbf{h}$, Confocal microscopy image of the DEA's cross section. The elastomer sheet is $220 \mu \mathrm{m}$ thick and it has seven elastomer layers. The thickness of the top and the bottom layers are approximately $65 \%$ of the middle layers. Scale bar represents $100 \mu \mathrm{m}$. i, Fabrication of the DEA. After the elastomer sheet is rolled into a cylindrical shell, the top and bottom cap are glued onto the DEA. A weight is placed on top of the DEA as the glue cures. After the glue cures, the DEA connections remain in compression (red) while the rest of the DEA returns to its neutral state (green). j, Front view of a DEA. Scale bar represents $3 \mathrm{~mm}$. 


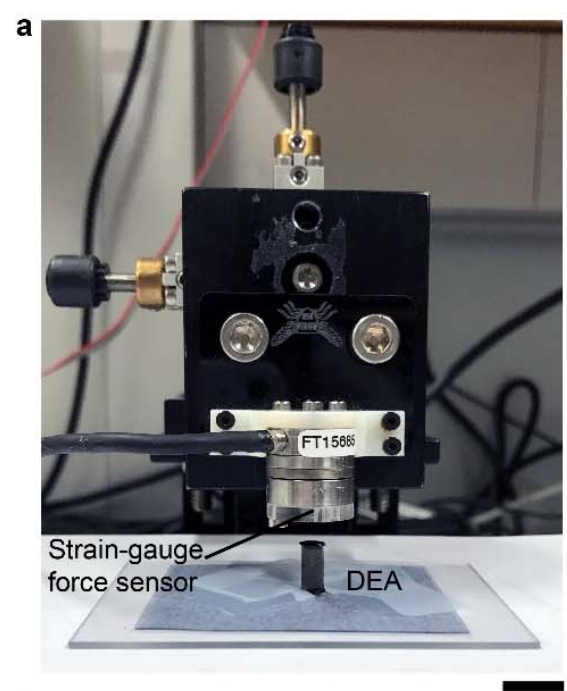

e

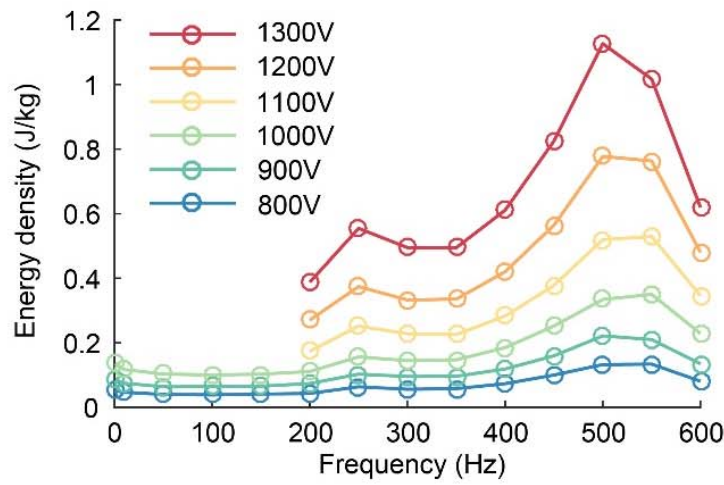

g
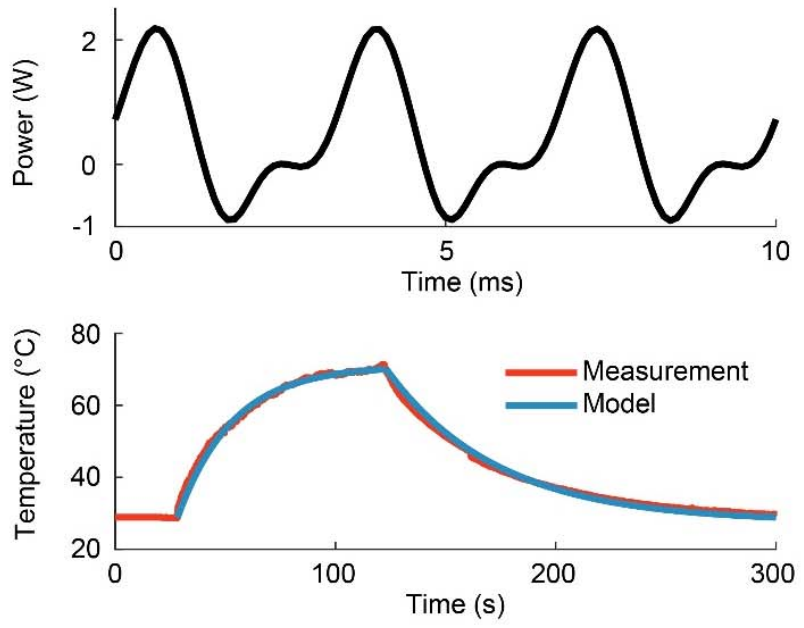
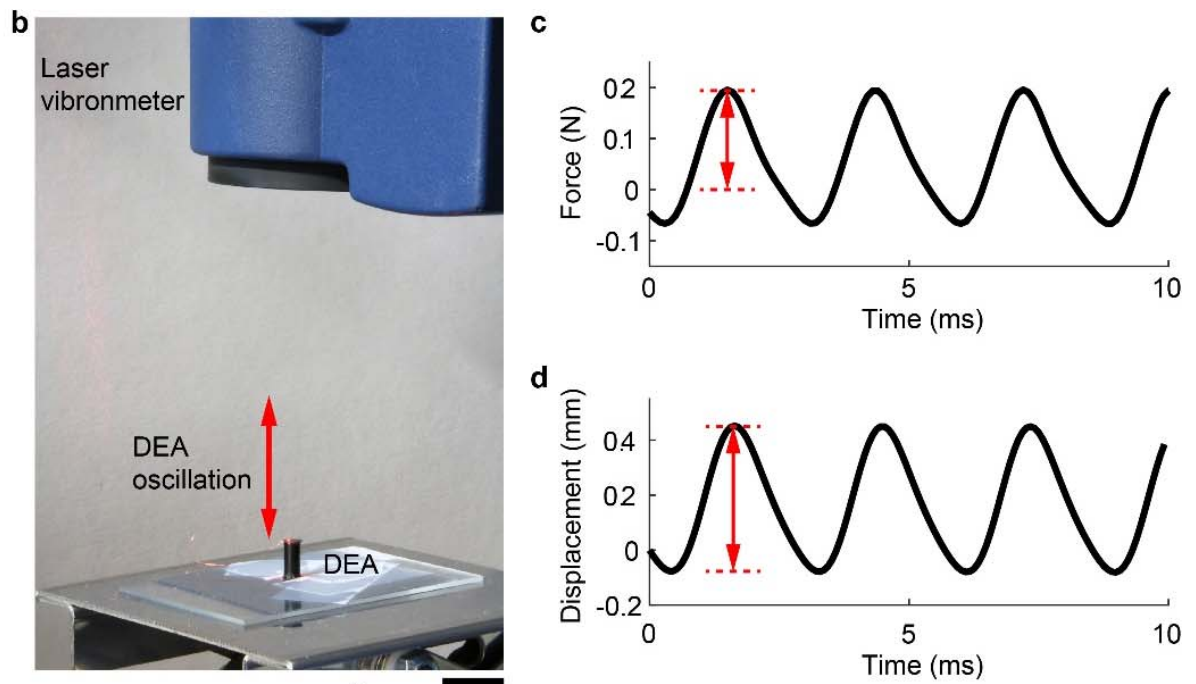

f
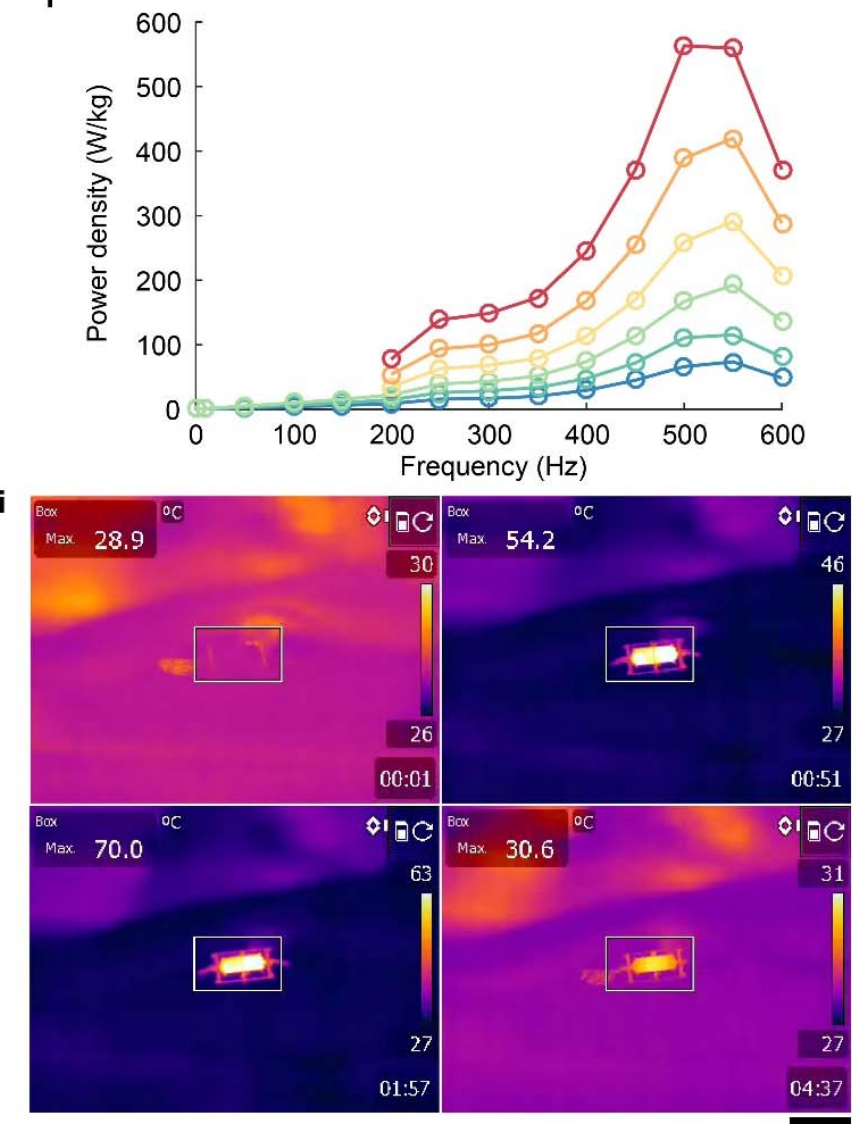

Extended Data Figure 2 | Characterization of blocked force, free displacement, and power dissipation. a, Experimental setup for measuring the DEA's blocked force. b, Experimental setup for measuring the DEA's free displacement. c-d, Sample blocked force (c) and free displacement (d) measurements when the DEA is driven at $350 \mathrm{~Hz}$ and $1300 \mathrm{~V}$. The red arrows in $\mathbf{c}$ and $\mathbf{d}$ indicate the ranges of blocked force and free displacement that correspond to Figure $2 \mathrm{a}$ and b. e-f, The DEA's energy (e) and power (f) density as functions of driving frequency and voltage. This DEA's blocked force and free displacement measurements are shown in Figure 2a-b. g, The DEA's instantaneous power consumption when driven at $1400 \mathrm{~V}$ and $300 \mathrm{~Hz}$. h, Measurement and modeling of the DEA's temperature profile during its operation at $1400 \mathrm{~V}$ and $300 \mathrm{~Hz}$. i, Thermal images showing the temperature of the DEA during operation. $\mathbf{h}$ and $\mathbf{i}$ show the same experiment. Scale bars in $(\mathbf{a}, \mathbf{b}$, and $\mathbf{i})$ represent $1 \mathrm{~cm}$. 

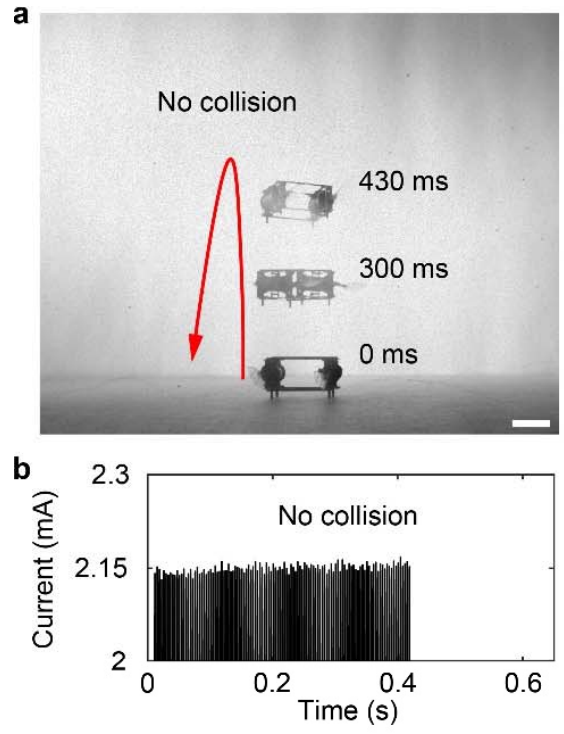

g

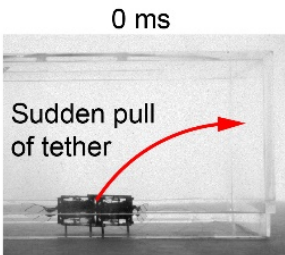

i

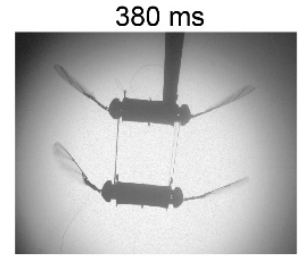

$420 \mathrm{~ms}$ c

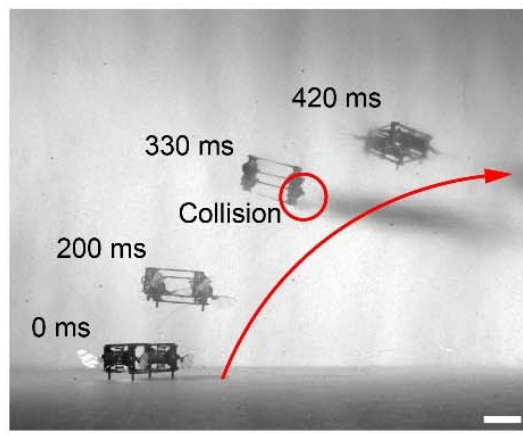

d
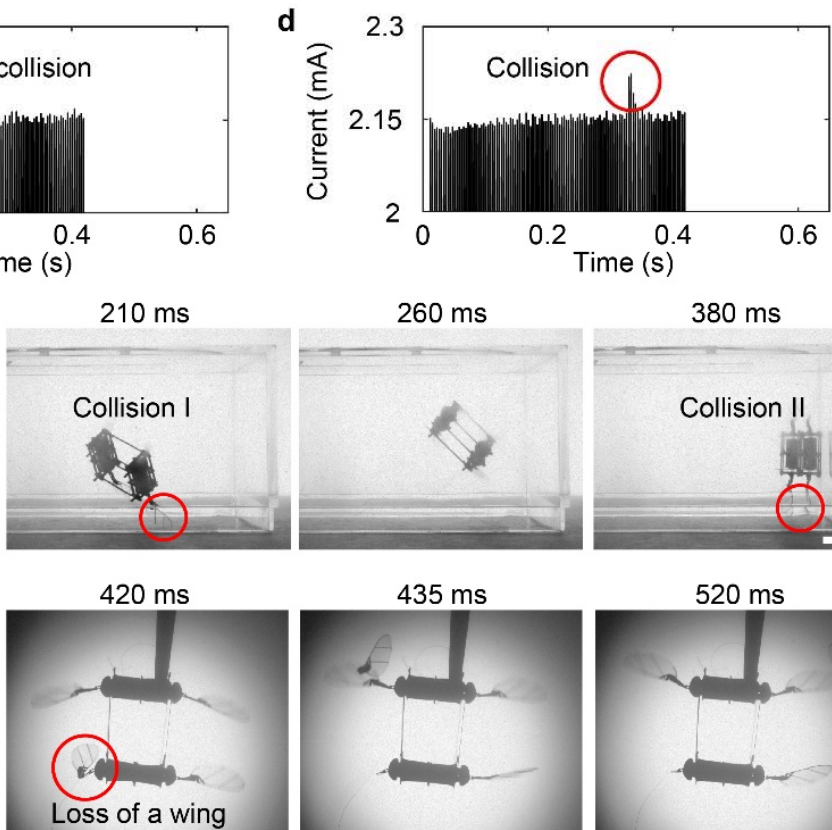

$260 \mathrm{~ms}$

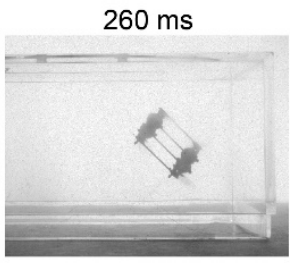

$435 \mathrm{~ms}$

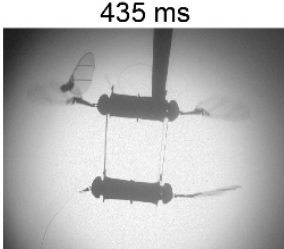

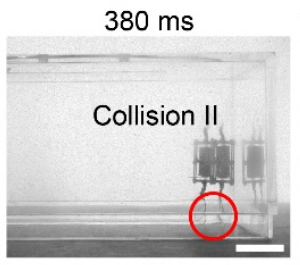

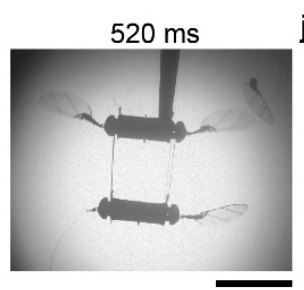

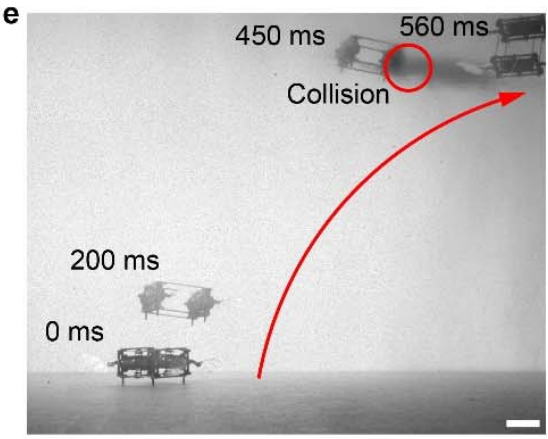
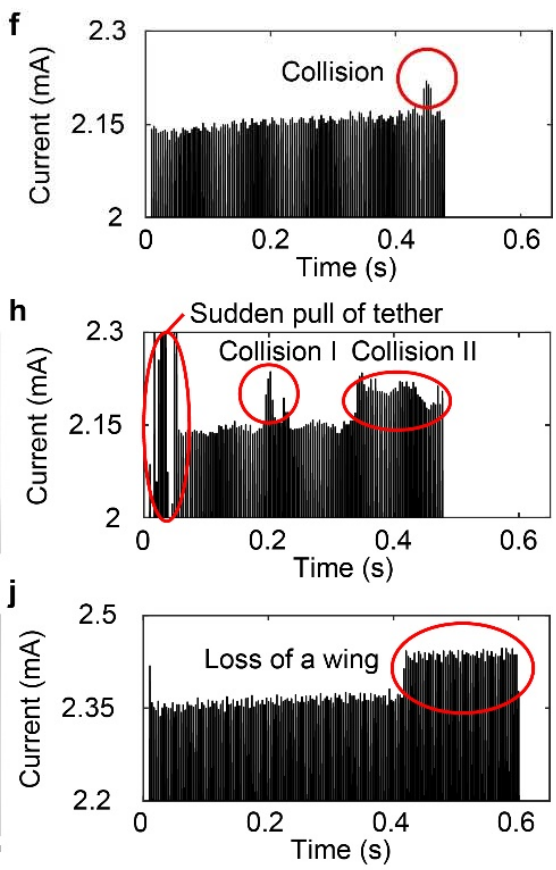

Extended Data Figure 3 | Robot in-flight collision and damage sensing. a-b, A composite image (a) and the measured DEA current (b) of a short takeoff flight without any collisions. c-f, Two takeoff flights in which the robot hits a wall during its ascent. The red circles in $\mathbf{c}$ and $\mathbf{e}$ mark the collision events and they correspond to the current spikes in $\mathbf{d}$ and $\mathbf{f}$, respectively. $\mathbf{g}-\mathbf{h}$, A robot takeoff flight in a transparent box. The robot makes multiple collisions and the red circles in $\mathbf{g}$ and $\mathbf{h}$ relate these collisions to DEA current changes. $\mathbf{i}-\mathbf{j}$, An image sequence (i) and the measured current (j) of a flapping-wing characterization test. One robot wing falls off during the experiment and this event is detected by measuring the DEA current. Scale bars in (a, c, e, g, i) represent $1 \mathrm{~cm}$. 


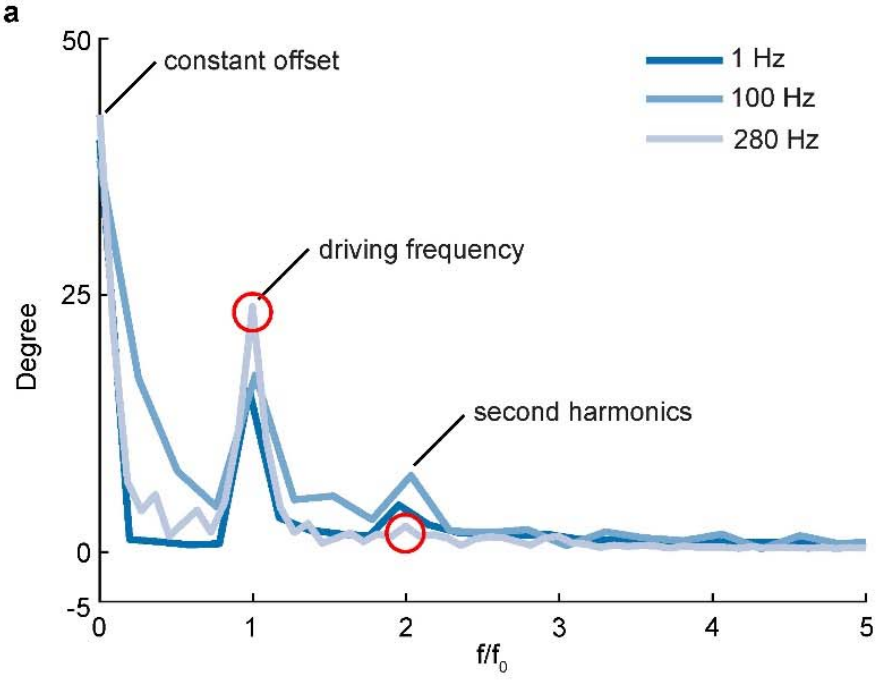

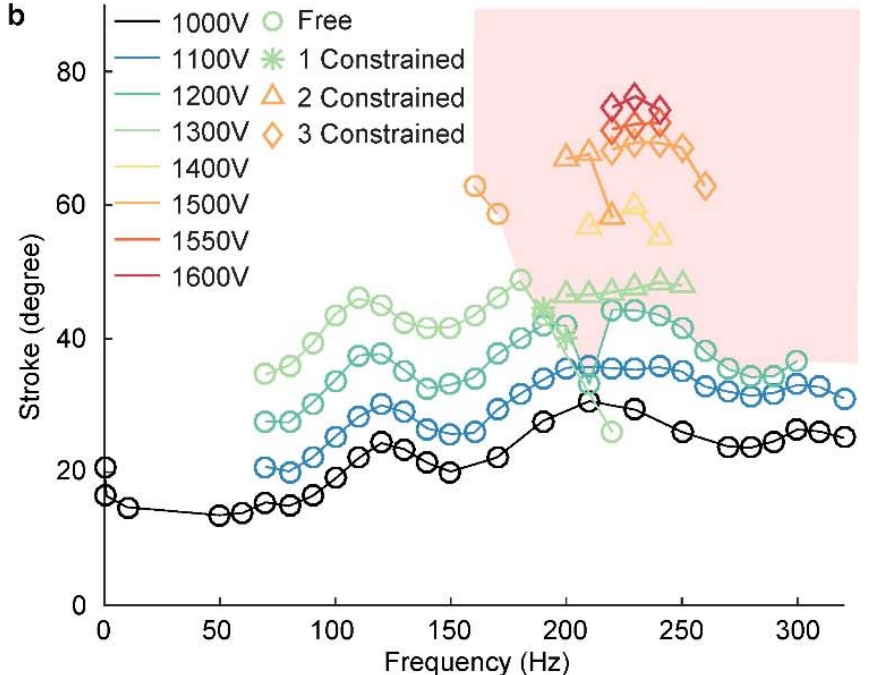

b

593

594 Extended Data Figure 4 | DEA actuation nonlinearity. a, Fast Fourier Transform of the tracked wing 595 stroke kinematics when a wing is driven at $1 \mathrm{~Hz}, 100 \mathrm{~Hz}$, and $280 \mathrm{~Hz}$. The stroke kinematics data is taken 596 from that shown in Figure 3b. There is a substantial second order harmonic for the cases of $1 \mathrm{~Hz}$ and 100 $\mathrm{Hz}$. When the wing is driven near the system resonant frequency $(280 \mathrm{~Hz})$, the red circles indicate that the 598 fundamental harmonic grows and the second harmonic is attenuated. $\mathbf{b}$, Right wing stroke amplitude as a 599 function of driving voltage and frequency. The red region represents stroke amplitudes and frequencies 600 that cannot be achieved without constraining the DEA. This data corresponds to the same experiment 601 shown in Figure 3f. 


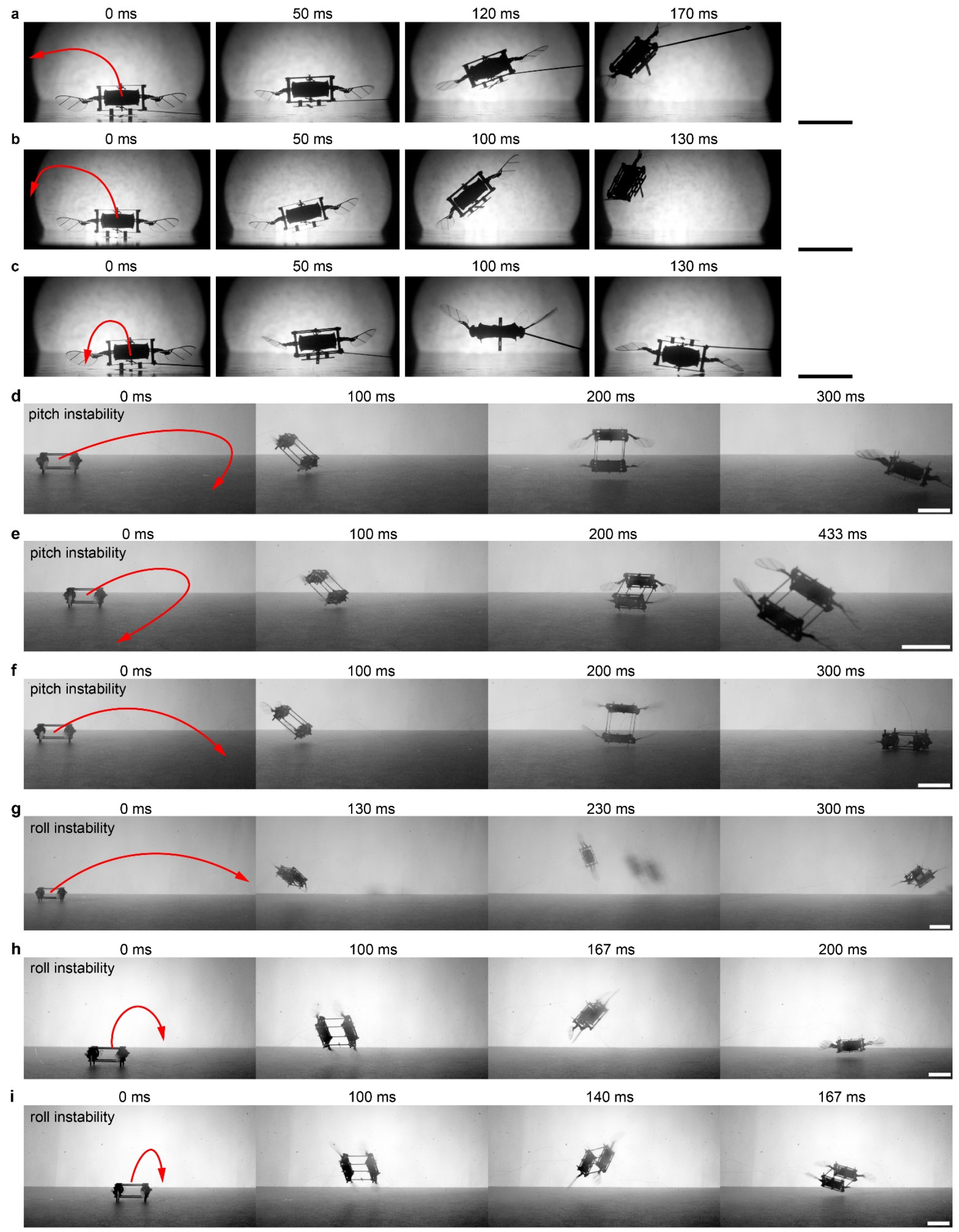


604 Extended Data Figure 5 | Repeated unstable takeoff flights. (a-c), Three takeoff flights of a robot with 605 one DEA. In these flights, the robot flips upside down within $200 \mathrm{~ms}$ after liftoff due to aerodynamic 606 torque imbalances from the two wings. (d-i), Unstable takeoff flights of a robot with two DEAs. In (d-f), 607 the robot pitches forward and eventually flips over due to asymmetric lift forces from the front and the 608 back robot modules. In (g-i), the robot rolls sideways and flips over due to lift force imbalances between 609 its left and right wings. Scale bars in (a-i) represent $1 \mathrm{~cm}$. 


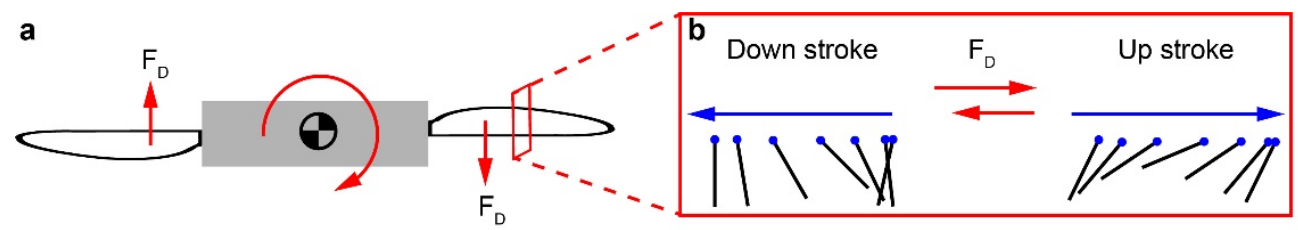

c

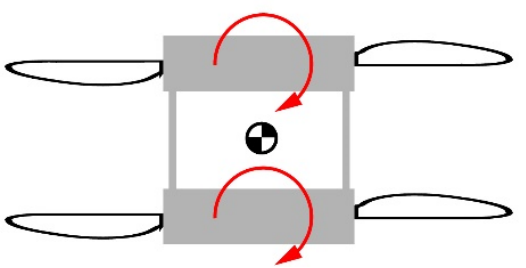

d

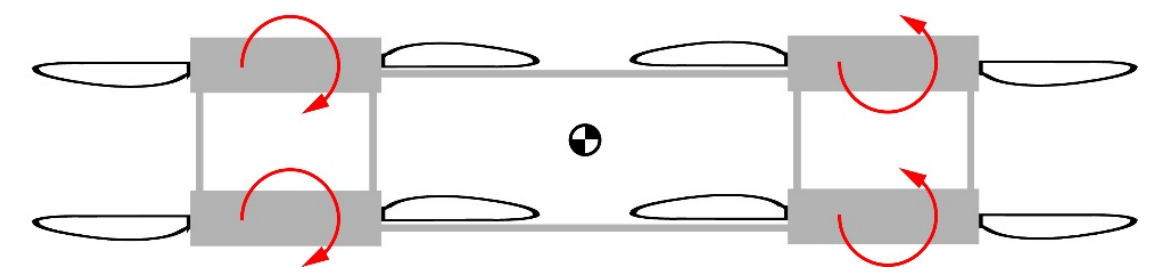

612 Extended Data Figure 6 | Illustration of robot yaw torque generation through biasing the mean wing 613 pitch angle. a, Illustration of wing pitch bias in an one-DEA module. The red arrows indicate the 614 directions of the mean drag force due to biasing the wing pitch. The net drag forces from the two wings 615 induce a robot yaw torque. $\mathbf{b}$, The inset shows the motion of a wing chord on a $2 \mathrm{D}$ plane. The wing pitch 616 bias causes different wing pitching motion in the up stroke and down stroke phases of the wing motion, 617 which leads to different drag forces. c, Two one-DEA modules having the same yaw torque bias direction 618 are assembled into a two-DEA robot. d, Two two-DEA modules having opposite yaw torque bias 619 directions are assembled into a four-DEA robot. 


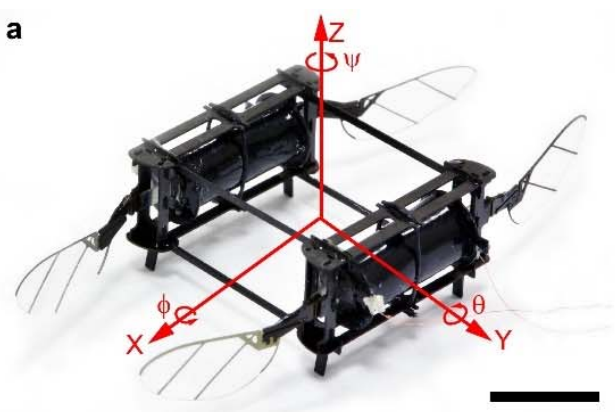

b

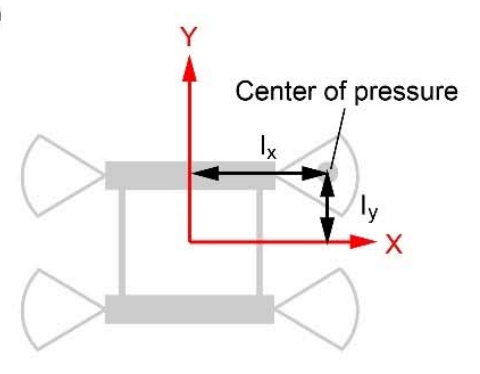

d
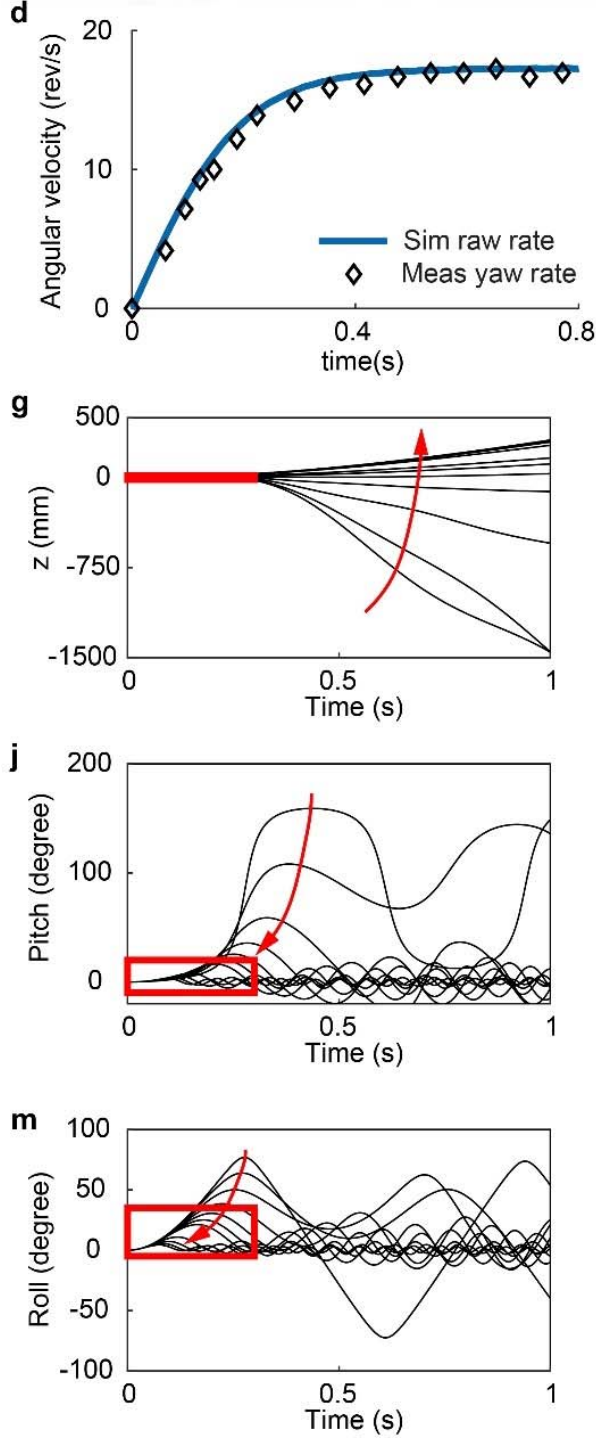

e

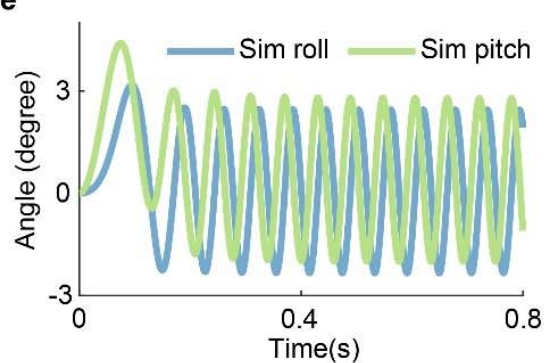

h
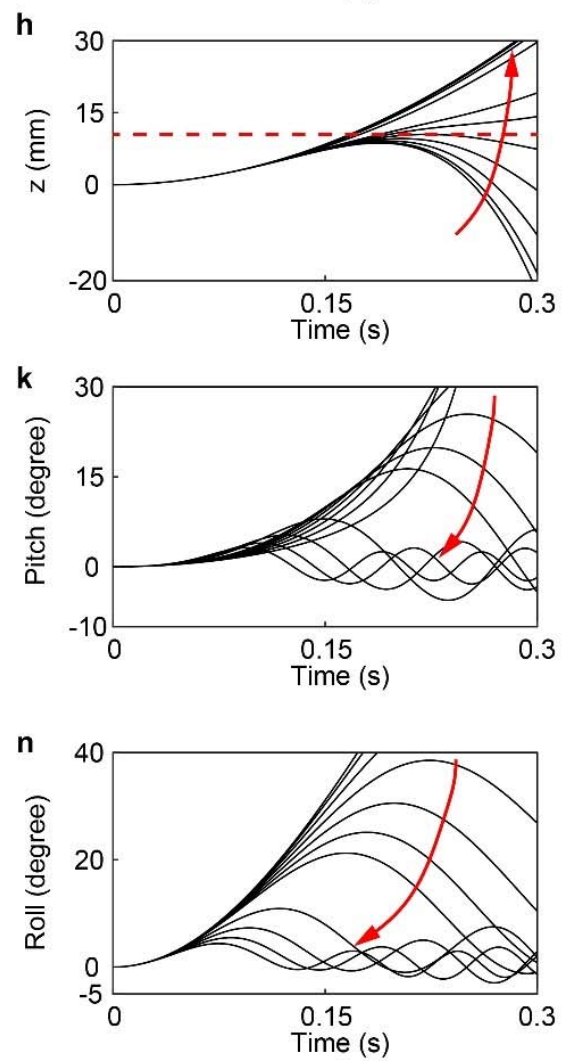

c

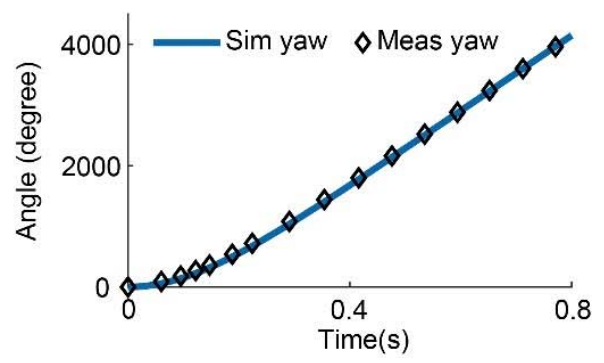

f

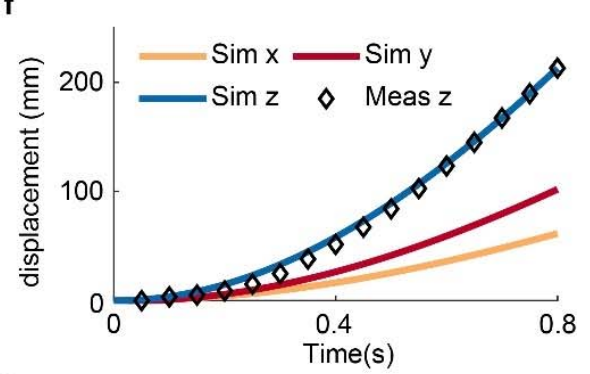

i

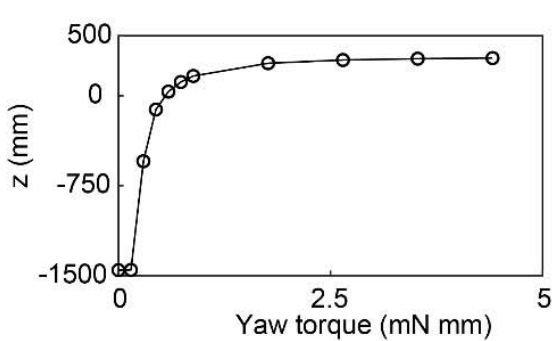

I
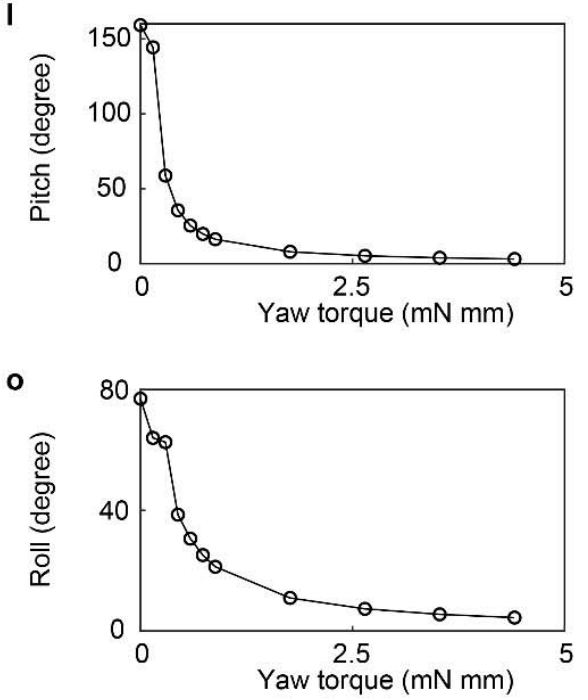

Extended Data Figure 7 | Simulation of open-loop ascending flight and comparison with experimental results. a, Coordinate system definition of the four-wing robot model. Scale bar represents $1 \mathrm{~cm}$. b, Top view schematic of the four-wing robot. $l_{x}$ and $l_{y}$ denote the distance from the robot's center of mass to each wing's center of pressure. c, Comparison of measured and simulated yaw $(\psi)$ motion. The robot makes 11 revolutions with respect to its z-axis $0.8 \mathrm{~s}$ after takeoff. $\mathbf{d}$, Comparison of measured and simulated yaw rate $(\dot{\psi})$. The steady state angular velocity of the robot's yaw rate is 17.5 revolutions per second. e, Simulated roll $(\phi)$ and pitch $(\theta)$ motion. Our simulation predicts that the steady state oscillation with respect to the robot's $X$ and $Y$ axes is smaller than $3^{\circ}$.f, Simulation results of the robot's displacement 
629 after takeoff. The experimental measurement of the robot's vertical motion is superimposed on the same 630 graph. The data shown in (c-f) correspond to the same simulation and experiment shown in Supplementary 631 Video 5 and Figure 4b-c. g-o, Dynamical simulation of robot takeoff flights under different values of body 632 yaw torque. g, Robot altitude as a function of time. h, A zoomed-in plot of robot altitude shortly after 633 takeoff. This plot corresponds to the red region in g. i, Robot altitude at one second after takeoff as a 634 function of input body yaw torque. $\mathbf{j}$, Robot pitch motion as a function of time. $\mathbf{k}$, A zoomed-in plot of 635 robot pitch that corresponds to the red region in j. l, Maximum robot pitch deviation as a function of input 636 yaw torque. $\mathbf{m}$, Robot roll motion as a function of time. $\mathbf{n}$, A zoomed-in plot of robot roll that corresponds 637 to the red region in $\mathbf{m}$. o, Maximum robot roll deviation as a function of input yaw torque. 

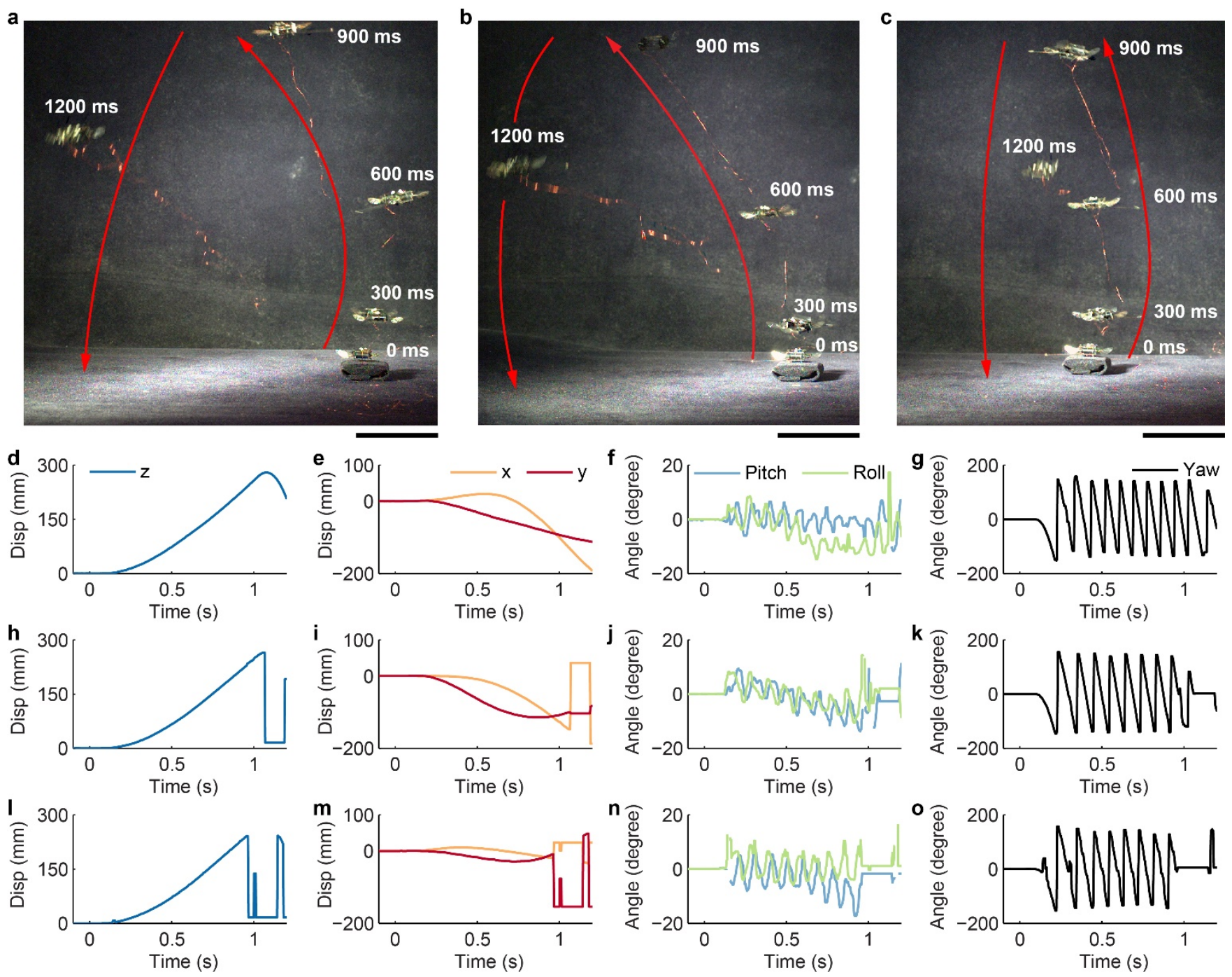

639

Extended Data Figure 8 | Three passively stable ascending flights of a robot with two DEAs. a-c,

641 Composite images of three one-second, open-loop ascending flights. d-g, Tracked robot altitude (d), $x$ 642 and y center of mass position (e), pitch and roll orientation (f), and yaw rotation (g). The data shown in $\mathbf{d}$ 643 g correspond to the flight shown in a. Similarly, (h-k) and (l-o) show the tracked flight data corresponding 644 to the flights shown in $\mathbf{b}$ and $\mathbf{c}$, respectively. Sudden jumps in the tracking data $(\mathbf{h}, \mathbf{i}, \mathbf{l}$, and $\mathbf{m})$ indicate 645 the time at which the Vicon motion capture system loses tracking. 
a

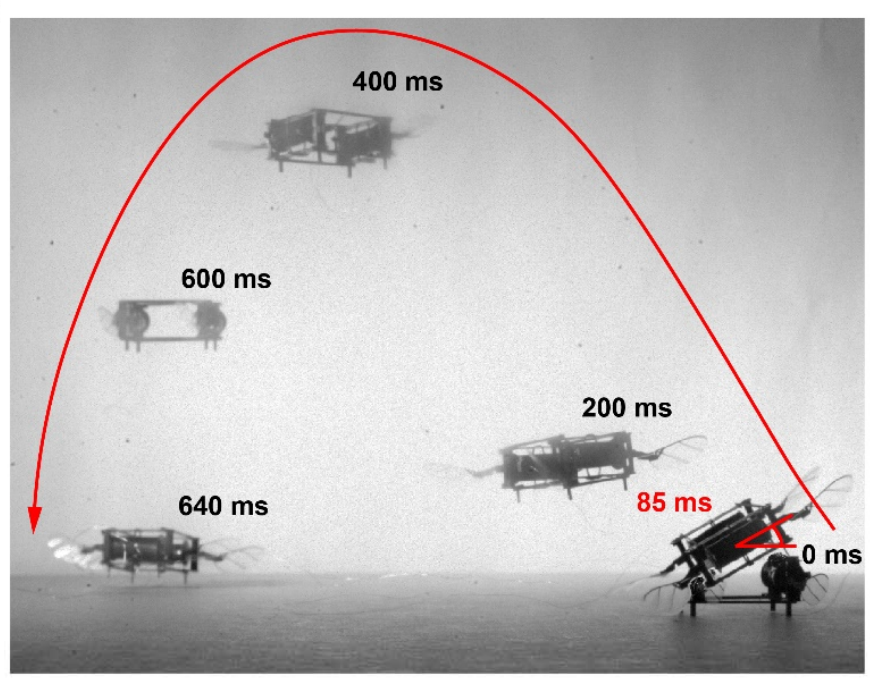

c

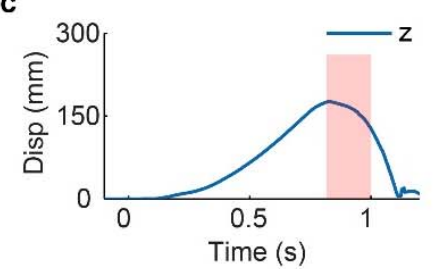

e

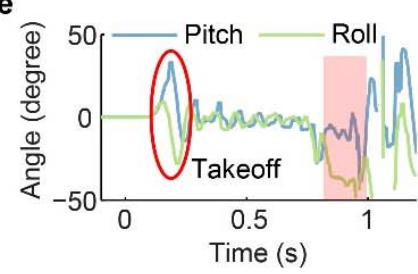

d

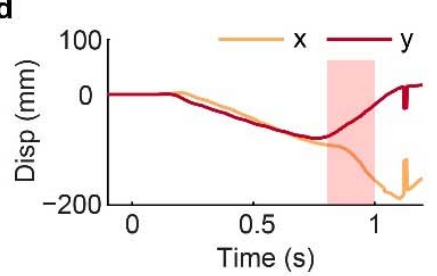

f

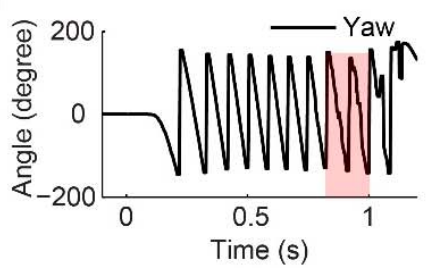

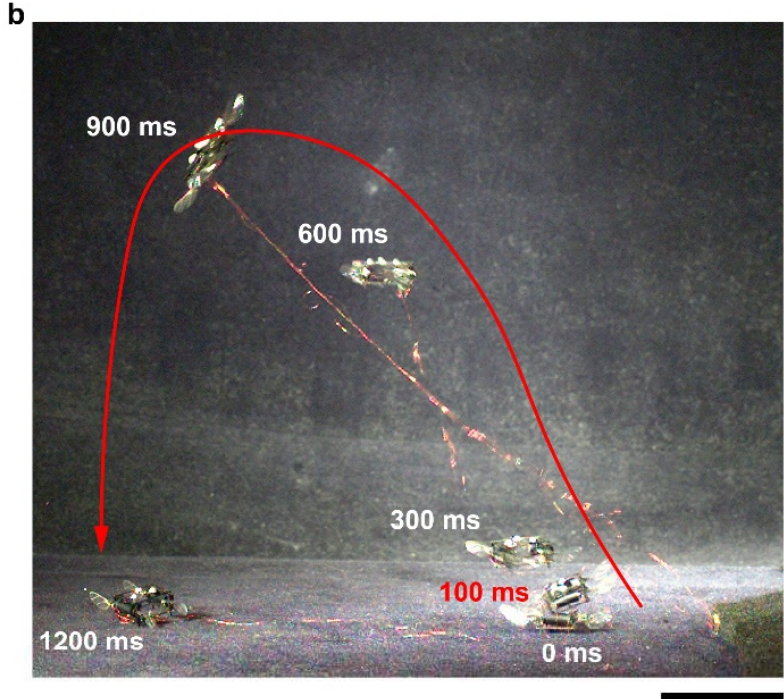

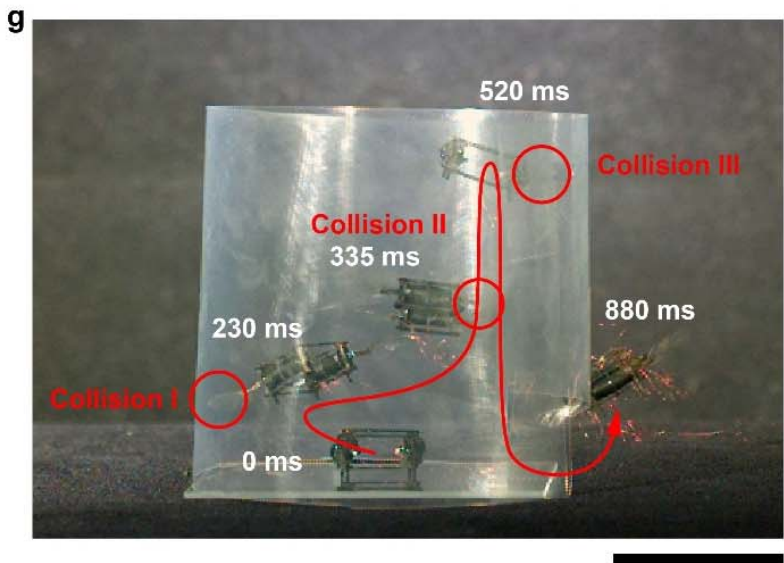

648

649

650

651

652

653

654

655

656

657

Extended Data Figure 9 | Robot unbalanced takeoff flights and a failed collision recovery experiment. a, A composite image of a 0.5 second open-loop takeoff flight captured at 3000 frames per second. The robot pitch deviation is approximately 35 degree $85 \mathrm{~ms}$ after takeoff. $\mathbf{b}$, A composite image of a 1 second open-loop takeoff flight conducted in the Vicon motion tracking arena. c-f, Tracked robot altitude (c), $\mathrm{x}$ and $\mathrm{y}$ center of mass position $(\mathbf{d})$, pitch and roll orientation $(\mathbf{e})$, and yaw rotation (f). The data shown in $\mathbf{c - f}$ correspond to the flight in $\mathbf{b}$. The red circle in $\mathbf{e}$ illustrates the large robot pitch and roll deviation after takeoff. The red shaded region in c-f show the changes of robot position and orientation after it is pulled by its tether. g, An example of a failed collision recovery experiment. The robot is destabilized after making the third collision. Scale bars in $\mathbf{a}, \mathbf{b}$, and $\mathbf{g}$ represent $5 \mathrm{~cm}$. 
a

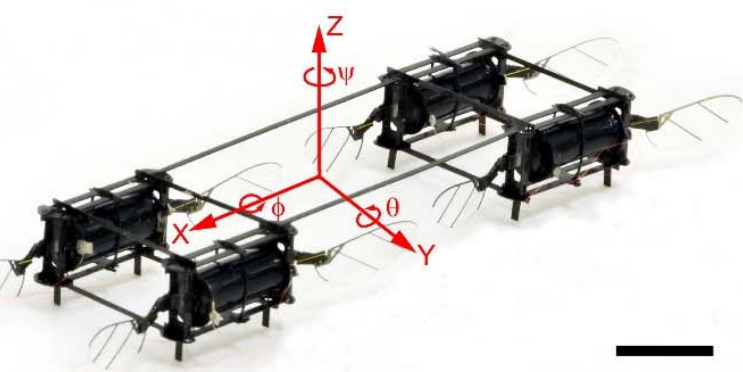

C

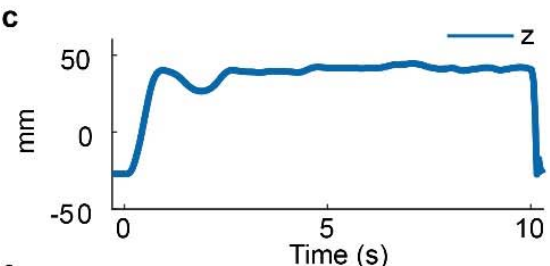

f
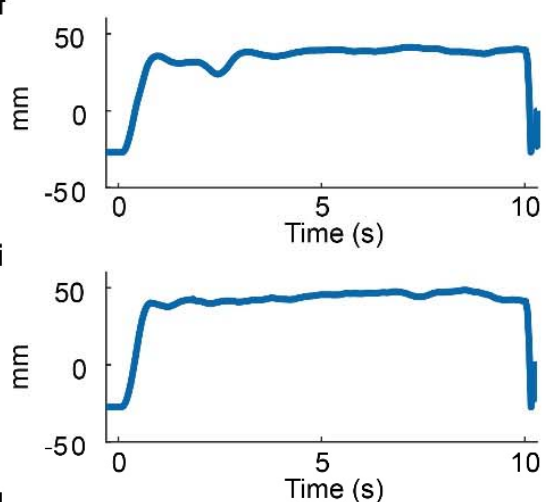

I

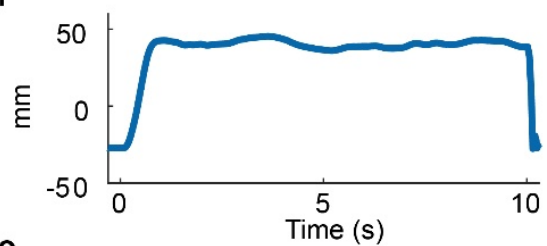

0

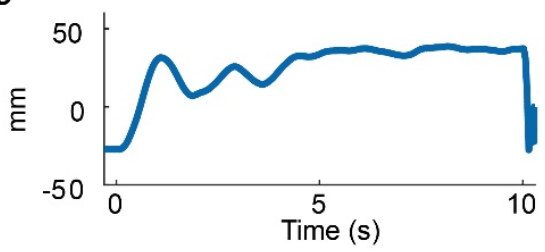

d

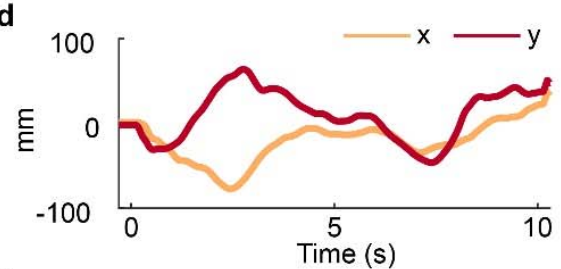

g
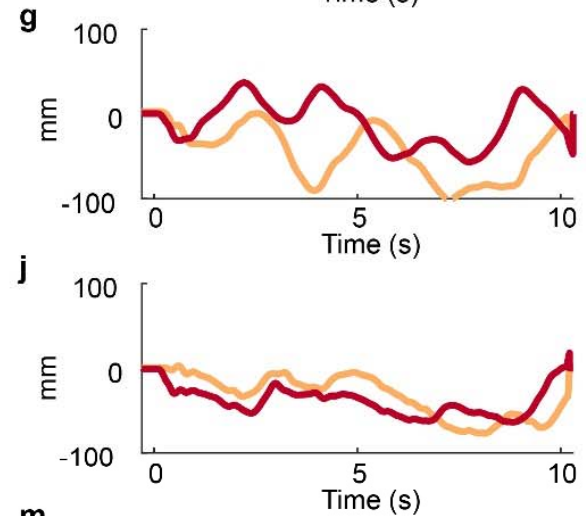

m
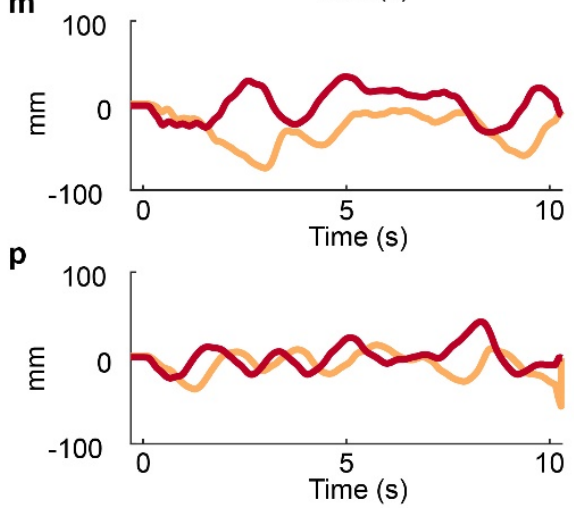

b

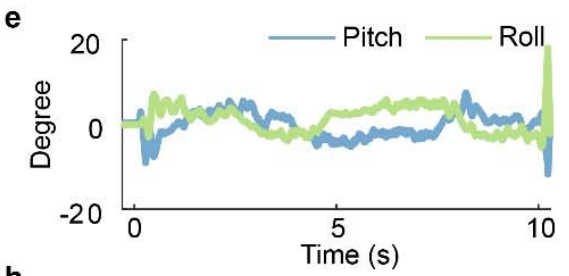

$\mathbf{h}$

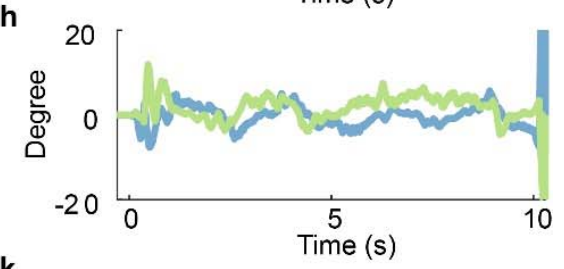

k

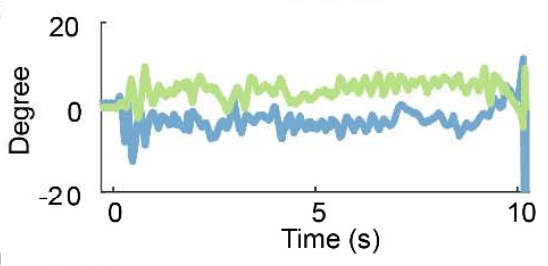

n
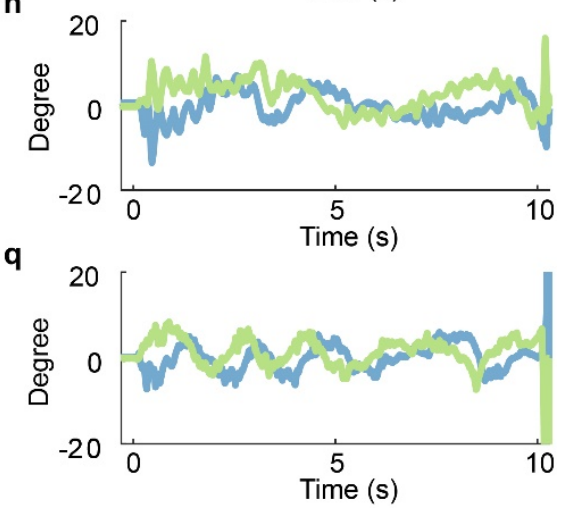

659

660

661

662

663

664

665

666

667

668

669

Extended Data Figure 10 | Controller design of the eight-wing robot and hovering flight repeatability. a, Perspective view of the eight-wing robot with a superimposed coordinate system. The roll $(\phi)$, pitch $(\theta)$, and yaw $(\psi)$ angles are defined with respect to the fixed $X, Y$, and $Z$ axes. Scale bar represents $1 \mathrm{~cm}$. b, Top view schematic of the eight-wing robot. $l_{x}$ and $l_{y}$ denote the distance from the robot center of mass to the geometric center of each DEA. c-q, Tracked robot position and attitude data of five 10-second hovering flights. In these flights, we do not control the robot's yaw motion. (c, f, i, l, o), The first column shows the robot's altitude as a function of time. $(\mathbf{d}, \mathbf{g}, \mathbf{j}, \mathbf{m}, \mathbf{p})$, The second column shows the robot's lateral position as a function of time. $(\mathbf{e}, \mathbf{h}, \mathbf{k}, \mathbf{n}, \mathbf{q})$, The last column shows the robot's roll $(\phi)$ and pitch $(\theta)$ motion as a function of time. 
671 Extended Data Table 1 | Parameters for the conceptual design of the two-wing robot.

\begin{tabular}{|c|c|c|}
\hline Parameter & Symbol & Value \\
\hline Robot mass & $m$ & $160 \mathrm{mg}$ \\
\hline Mean drag coefficient & $\overline{C_{D}}$ & 1.6 \\
\hline Mean lift coefficient & $\overline{C_{L}}$ & 0.7 \\
\hline Transmission ratio & $T$ & $2530 \mathrm{rad} \cdot \mathrm{m}^{-1}$ \\
\hline Maximum lift to weight ratio & $f_{m}$ & 1.2 \\
\hline Robot transmission dimensions & $t, w, l$ & $25 \mu \mathrm{m}, 1.2 \mathrm{~mm}, 200 \mu \mathrm{m}$ \\
\hline Young's modulus of polyimide film & $E$ & $2.5 \mathrm{GPa}$ \\
\hline Wing aspect ratio & $A R$ & 3 \\
\hline Wing span & $R$ & $9.9 \mathrm{~mm}$ \\
\hline Wing span wise moment of inertia & $I_{z z}$ & $15 \mathrm{mg} \cdot \mathrm{mm}^{2}$ \\
\hline Wing span wise center of pressure & $r_{c p}$ & $7 \mathrm{~mm}$ \\
\hline Air density & $\rho$ & $1.2 \mathrm{~kg} \cdot \mathrm{m}^{-3}$ \\
\hline Wing hinge geometry & $t_{h}, w_{h}, l_{h}$ & $7.5 \mu \mathrm{m}, 2.65 \mathrm{~mm}, 110 \mu \mathrm{m}$ \\
\hline DEA mass & $m_{a}$ & $100 \mathrm{mg}$ \\
\hline DEA natural resonance frequency & $f_{\text {res }}$ & $465 \mathrm{~Hz}$ \\
\hline Heat generated during operation & $Q$ & $0.25 \mathrm{~J} \cdot \mathrm{s}^{-1}$ \\
\hline DEA heat capacity & C & $0.15 \mathrm{~J} \cdot \mathrm{K}^{-1}$ \\
\hline Ambient temperature & $T_{a}$ & $28.7^{\circ} \mathrm{C}$ \\
\hline Initial temperature at onset of cooling & $T_{i}$ & $70{ }^{\circ} \mathrm{C}$ \\
\hline Heat conduction rate during heating & $K_{1}$ & $0.04 \mathrm{~s}^{-1}$ \\
\hline Heat conduction rate during cooling & $K_{2}$ & $0.022 \mathrm{~s}^{-1}$ \\
\hline
\end{tabular}


673 Extended Data Table 2 | Physical and simulation parameters for the four-wing robot. These parameter 674 values correspond to the simulation results shown in Extended Data Figure 7.

\begin{tabular}{|c|c|c|}
\hline Parameter & Symbol & Value \\
\hline Mass & $m$ & $320 \mathrm{mg}$ \\
\hline Principal moment of inertia & $I_{x x}, I_{y y}, I_{z z}$ & $\begin{array}{c}2.99 \times 10^{4}, 2.41 \times 10^{3}, \\
3.13 \times 10^{4} \mathrm{mg} \cdot \mathrm{mm}^{2}\end{array}$ \\
\hline Distance to robot center of mass & $l_{x}, l_{y}$ & $13.3,7 \mathrm{~mm}$ \\
\hline Lift force of each wing & $F_{L 1}, F_{L 2}, F_{L 3}, F_{L 4}$ & $0.86,0.81,0.82,0.88 \mathrm{mN}$ \\
\hline Drag force of each wing & $F_{D 1}, F_{D 2}, F_{D 3}, F_{D 4}$ & $0.29,0.29,0.29,0.29 \mathrm{mN}$ \\
\hline Body damping force coefficient & $b_{f}$ & $0.5 \mathrm{mg} \cdot \mathrm{mm}{ }^{-1}$ \\
\hline Body damping torque coefficient & $b_{t}$ & $1.5 \times 10^{3} \mathrm{mg} \cdot \mathrm{mm}^{2}$ \\
\hline
\end{tabular}


676 Extended Data Table 3 | Values of flight controller parameters for hovering flights corresponding to 677 Figure 4e-i and Extended Data Figure 10.

\begin{tabular}{|c|c|c|c|c|c|c|c|c|c|c|}
\hline$\#$ & $\begin{array}{c}\text { Flight } \\
\text { duration } \\
(\mathrm{s})\end{array}$ & $\begin{array}{c}\lambda_{0} \\
\left(\mathrm{~s}^{-4}\right)\end{array}$ & $\begin{array}{c}\lambda_{1} \\
\left(\mathrm{~s}^{-3}\right)\end{array}$ & $\begin{array}{c}\lambda_{2} \\
\left(\mathrm{~s}^{-2}\right)\end{array}$ & $\begin{array}{c}\lambda_{3} \\
\left(\mathrm{~s}^{-1}\right)\end{array}$ & $\begin{array}{c}\Lambda_{0} \\
\left(\mathrm{~s}^{-2}\right)\end{array}$ & $\begin{array}{c}\Lambda_{1} \\
\left(\mathrm{~s}^{-1}\right)\end{array}$ & $\begin{array}{c}\alpha \\
(\mathrm{V} / \mathrm{mN})\end{array}$ & $\begin{array}{c}\beta \\
(\mathrm{V})\end{array}$ & $\begin{array}{c}\gamma_{1}, \gamma_{2}, \gamma_{3}, \gamma_{4} \\
(\mathrm{~V})\end{array}$ \\
\hline 1 & 10 & 13608 & 6631 & 798 & 62 & 25 & 125 & 82 & 1172 & $38,80,69,34$ \\
\hline 2 & 10 & 13608 & 6631 & 798 & 62 & 25 & 125 & 82 & 1172 & $38,80,69,34$ \\
\hline 3 & 10 & 27216 & 9946 & 570 & 57 & 25 & 125 & 82 & 1172 & $38,80,69,34$ \\
\hline 4 & 10 & 30618 & 9946 & 570 & 57 & 25 & 125 & 82 & 1172 & $38,80,69,34$ \\
\hline 5 & 10 & 54432 & 13262 & 713 & 71 & 25 & 125 & 82 & 1172 & $38,80,69,34$ \\
\hline 6 & 16 & 54432 & 13262 & 713 & 71 & 30 & 150 & 82 & 1172 & $38,85,74,34$ \\
\hline
\end{tabular}

